\title{
FLEXRENDER: A DISTRIBUTED RENDERING ARCHITECTURE FOR RAY TRACING HUGE SCENES ON COMMODITY HARDWARE.
}

\author{
A Thesis \\ Presented to \\ the Faculty of California Polytechnic State University \\ San Luis Obispo
}

\author{
In Partial Fulfillment \\ of the Requirements for the Degree \\ Master of Science in Computer Science
}

by

Robert Edward Somers

June 2012 
(C) 2012

Robert Edward Somers

ALL RIGHTS RESERVED 


\section{COMMITTEE MEMBERSHIP}

TITLE:

FlexRender: A distributed rendering architecture for ray tracing huge scenes on commodity hardware.

AUTHOR: $\quad$ Robert Edward Somers

DATE SUBMITTED: June 2012

COMMITTEE CHAIR: Z Zö̈ Wood, Ph.D.

COMMITTEE MEMBER: Chris Lupo, Ph.D.

COMMitTeE MEMBER: Phillip Nico, Ph.D. 


\begin{abstract}
FlexRender: A distributed rendering architecture for ray tracing huge scenes on commodity hardware.

Robert Edward Somers
\end{abstract}

As the quest for more realistic computer graphics marches steadily on, the demand for rich and detailed imagery is greater than ever. However, the current "sweet spot" in terms of price, power consumption, and performance is in commodity hardware. If we desire to render scenes with tens or hundreds of millions of polygons as cheaply as possible, we need a way of doing so that maximizes the use of the commodity hardware we already have at our disposal.

Techniques such as normal mapping and level of detail have attempted to address the problem by reducing the amount of geometry in a scene. This is problematic for applications that desire or demand access to the scene's full geometric complexity at render time. More recently, out-of-core techniques have provided methods for rendering large scenes when the working set is larger than the available system memory.

We propose a distributed rendering architecture based on message-passing that is designed to partition scene geometry across a cluster of commodity machines in a spatially coherent way, allowing the entire scene to remain in-core and enabling the construction of hierarchical spatial acceleration structures in parallel. The results of our implementation show roughly an order of magnitude speedup in rendering time compared to the traditional approach, while keeping memory overhead for message queuing around $1 \%$. 


\section{Contents}

List of Tables viii

List of Figures $\quad$ ix

1 Introduction 1

1.1 Geometric Complexity . . . . . . . . . . . . . . . 1

1.2 Parallel Rendering . . . . . . . . . . . . . . . . . 2

1.3 Our Contribution ................... . . 4

2 Background $\quad 6$

2.1 Light and Radiometry . . . . . . . . . . . . . 6

2.2 Ray Tracing . . . . . . . . . . . . . . . . 8

2.3 Bounding Volume Hierarchies . . . . . . . . . . . . . . . . . . . . 10

2.4 Morton Coding and the Z-Order Curve . . . . . . . . . . . . . . . 12

3 Related Work 15

3.1 Big Geometry Workarounds . . . . . . . . . . . . . . . 16

3.1.1 Normal Mapping . . . . . . . . . . . . . 16

3.1 .2 Level of Detail . . . . . . . . . . . . . . . . . . . 17

3.1.3 Out-of-Core ................... 17

3.2 Parallel Rendering . . . . . . . . . . . . . . . . . 18

3.2.1 Cooperative Networked Rendering . . . . . . . . . 18

3.2.2 General Purpose GPU Rendering . . . . . . . . . . . . . . 19

3.3 MapReduce ....................... 20

4 FlexRender Architecture $\quad 22$

4.1 Organization and Design . . . . . . . . . . . . . . 23 
4.1.1 Workers and the Renderer . . . . . . . . . . . 23

4.1 .2 Fat Rays . . . . . . . . . . . . . . . . 24

4.1 .3 General Types . . . . . . . . . . . . . . . . 25

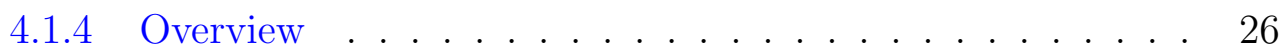

4.2 Render Preparation . . . . . . . . . . . . . . . . . . . 27

4.2.1 Configuration and Asset Distribution . . . . . . . . . . . 28

4.2.2 Parallel Construction of Spatial Acceleration Structures . . 29

4.3 Ray Processing . . . . . . . . . . . . . . . . . . . . 30

4.3.1 Ray Queues ... . . . . . . . . . . . . 32

4.3.2 Primary Ray Casting . . . . . . . . . . . . . . 33

4.3.3 Distributed BVH Traversal . . . . . . . . . . . . . . 34

4.3.4 Illumination ................... . . 40

4.3.5 Shading ..................... . . 42

4.4 Render Completion . . . . . . . . . . . . . . . . . . 44

4.4.1 Statistics and Monitoring ........... . 45

4.4 .2 Image Synthesis . . . . . . . . . . . . . . . . . . 46

5 Results $\quad 48$

5.1 Experimental Setup . . . . . . . . . . . . . . . . . . 48

5.2 Toy Store . . . . . . . . . . . . . . . . . . . . . . . 49

5.3 Render Time . . . . . . . . . . . . . . . . . . . . . . . 49

5.3.1 Traditional Method . . . . . . . . . . . . 50

5.3.2 FlexRender .................... 51

5.4 Geometry Distribution . . . . . . . . . . . . . . 52

5.5 Network Hops . . . . . . . . . . . . . . . . . 54

5.6 Ray Queue Sizes . . . . . . . . . . . . . . . . . . . 55

5.7 Cluster Size . . . . . . . . . . . . . . . . . 56

5.8 Example Renders . . . . . . . . . . . . . . . . . 57

5.9 Summary ............................... 61

6 Future Work $\quad 63$

6.1 System Optimizations . . . . . . . . . . . . . 63 
6.1.1 Eliminating Extra Network Hops . . . . . . . . . . . . . . 63

6.1.2 Competing Concerns ............... 64

6.2 Memory Optimizations . . . . . . . . . . . . . 66

6.3 GPGPU and Heterogeneous Architectures . . . . . . . . . . . 66

$\begin{array}{ll}\text { Bibliography } & 68\end{array}$ 


\section{List of Tables}

5.1 Time (in seconds) for individual workers to complete each phase using the traditional method. . . . . . . . . . . . . . . 51

5.2 Time (in seconds) for each phase of rendering with FlexRender and the traditional configuration. . . . . . . . . . . . . 52

5.3 Distribution of geometry and acceleration structure data based on storage requirements. . . . . . . . . . . . . . . . . 54

5.4 Percentage of rays that were processed by the given number of workers. . . . . . . . . . . . . . . . 54

5.5 Size of ray queues when rendering Toy Store with a 16-worker FlexRender cluster. . . . . . . . . . . . . . . . . . . 57

5.6 Comparison of cluster sizes with both the traditional configuration and the FlexRender configuration. . . . . . . . . . . . 58 


\section{List of Figures}

1.1 Typical memory latencies in a modern commodity machine. Gigabit Ethernet latency included for comparison. . . . . . . . . . . 3

2.1 Ray tracing models light by shooting rays into the scene from the camera. Credit: "Henrik" (Creative Commons License) . . . . . . 8

2.2 Reflection and refraction are modeled by casting new rays from intersection points. Credit: "Stannered" (Creative Commons License) 9

2.3 Bounding volume hierarchies partition primitives into a binary tree structure. Credit: "Schreiberx" (Creative Commons License) . . . 10

2.4 Examples of two dimensional and three dimensional Z-order curves. Credit: "David Eppstein" and "Robert Dickau" (Creative Commons License $)$. . . . . . . . . . . . . . . . . . .

3.1 Normal mapping uses a texture to store high resolution shading normals on low resolution geometry. . . . . . . . . . . . . . . 16

4.1 Workers maintain connections with all other workers in the cluster. The renderer does the same. . . . . . . . . . . . . . . . . . . . 24

4.2 The three different ray types and their interactions. . . . . . . . . 31

4.3 Example top-level BVH with 5 workers. . . . . . . . . . . . . . 34

4.4 The ray fails its first test and moves to the sibling node. . . . . . 35

4.5 Traversal down the left-hand nodes continues as long as bounding tests pass. . . . . . . . . . . . . . . . . . 35

4.6 The ray is sent to worker $\mathrm{C}$ and tested against local geometry for intersections. . . . . . . . . . . . . . 36

4.7 The ray is sent to worker $\mathrm{D}$ and again tested against local geometry for intersections. . . . . . . . . . . . . . . . . . . . . 37 
4.8 With successful hit record in hand, traversal continues back up the tree. . . . . . . . . . . . . . . . . . . 38

4.9 Worker E's bounding test passed, but was further away than the hit record. We arrive at the root and traversal is complete. . . . .

4.10 The left and right images show the geometry split between two workers. The composite image is in the center. Note how the Buddha reflects the left side geometry in the left image and the right side geometry in the right image. The actual Buddha mesh data was distributed to the worker on the left. . . . . . . . . . 47

5.1 The "Toy Store" scene used for benchmarking FlexRender. . . . . 50

5.2 Slices of the Toy Store scene rendered with 8 machines in the traditional configuration. . . . . . . . . . . . . . 51

5.3 The individual worker image buffers that are combined to form the final image. . . . . . . . . . . . . . . . 53

5.4 Percentage of total geometry that was distributed to each worker. 54

5.5 Number of rays queued on each worker over time. Different colors correspond to different workers. . . . . . . . . . . . . . 56

5.6 Toy Store scene that is comprised of nearly 1,300 models and 42 million triangles. . . . . . . . . . . . . . . . 58

5.7 Field of high resolution models with nearly 87 million triangles. . 59

5.8 A monochromatic Cornell Box, showing the distribution of geometry between two workers. . . . . . . . . . . . . . . 59

5.9 A Cornell Box with a toon shaded Stanford bunny and mirrored Buddha. . . . . . . . . . . . . . . . . . . 60

5.10 A Cornell Box with a Monte Carlo global illumination shader. The top row shows individual worker renders, the bottom center is the final composite. Each worker has direct light for the geometry on its machine and indirect light caused by the geometry on its machine. 61 


\section{Chapter 1}

\section{Introduction}

Rendering, the process of taking the description of a scene and turning it into a visual image, has advanced at an incredible pace in recent years. Many techniques have been developed to turn these descriptions into pixels, and countless more have targeted weaknesses or challenges with those algorithms. As the field continues to evolve and grow, new algorithms will be born, others will fall out of favor and die, and some will soldier on. Only one thing remains constant: The desire to bring our computational models ever closer to mimicking physical reality.

\subsection{Geometric Complexity}

At the heart of mimicking reality is describing the world we wish to show. In computer graphics, this has traditionally been done by defining surfaces. While exciting developments in volume rendering techniques happen on a regular basis, it is unlikely we will abandon using surfaces any time soon. Unfortunately for volumes, algorithms that operate on them are inherently $n^{3}$ (where $n$ is the size 
of the volume in one dimension) and $n^{3}$ is not a particularly friendly number in the field of Computer Science.

For now and the foreseeable future, it seems, surfaces will be our bread and butter. Many methods have been developed for describing surfaces in computer programs, such as level sets, implicitly defined surfaces from mathematical equations, and curvilinear forms such as parametric splines. The undisputed champion of surface representations, however, has been the polygonal mesh. Countless manyears of research and development has yielded efficient techniques for processing meshes of interconnected polygons at blistering speeds, and many other surface representations are ultimately converted to a polygonal mesh at some stage of the rendering pipeline.

Meshes are easy for artists to work with because they represent discrete points in space and the connectivity between those points (rather than abstract equations). However, their core advantage is also their core drawback. Because everything is defined explicitly, meshes with fine levels of detail have significantly higher storage requirements. Thus, as the demand for higher visual fidelity increases, the natural tendency is to increase geometric complexity.

\subsection{Parallel Rendering}

Graphics has long been said to be a problem that is embarrassingly parallel, given that many graphics algorithms operate on pixels independently. Graphics processing units (GPUs) have exploited this fact for many years to achieve amazing throughput of graphics primitives in real-time. Ray tracing in particular (discussed more thoroughly in Section 2.2) is commonly paraded as the poster child for parallel processing. 
Unfortunately the story is not all unicorns and rainbows. While processor architectures have become exceedingly parallel and posted impressive speedups, the memory hierarchy has not had time to catch up. For a processor to perform well, the CPI, cycles per instruction, must remain low to ensure time is spent doing useful work and not waiting on data.

In current memory hierarchies, data access time can take anywhere from around 12 cycles (4 nanoseconds for an L1 cache hit) to over 300 cycles (100 nanoseconds for main memory). Techniques such as out-of-order execution are helpful in filling this wasted time, but for memory intensive applications it can be difficult to fill all the gaps with useful work. Thus, keeping the chips "hot" by reducing time spent waiting on data is critical to achieve maximum performance, and is an extremely challenging problem.

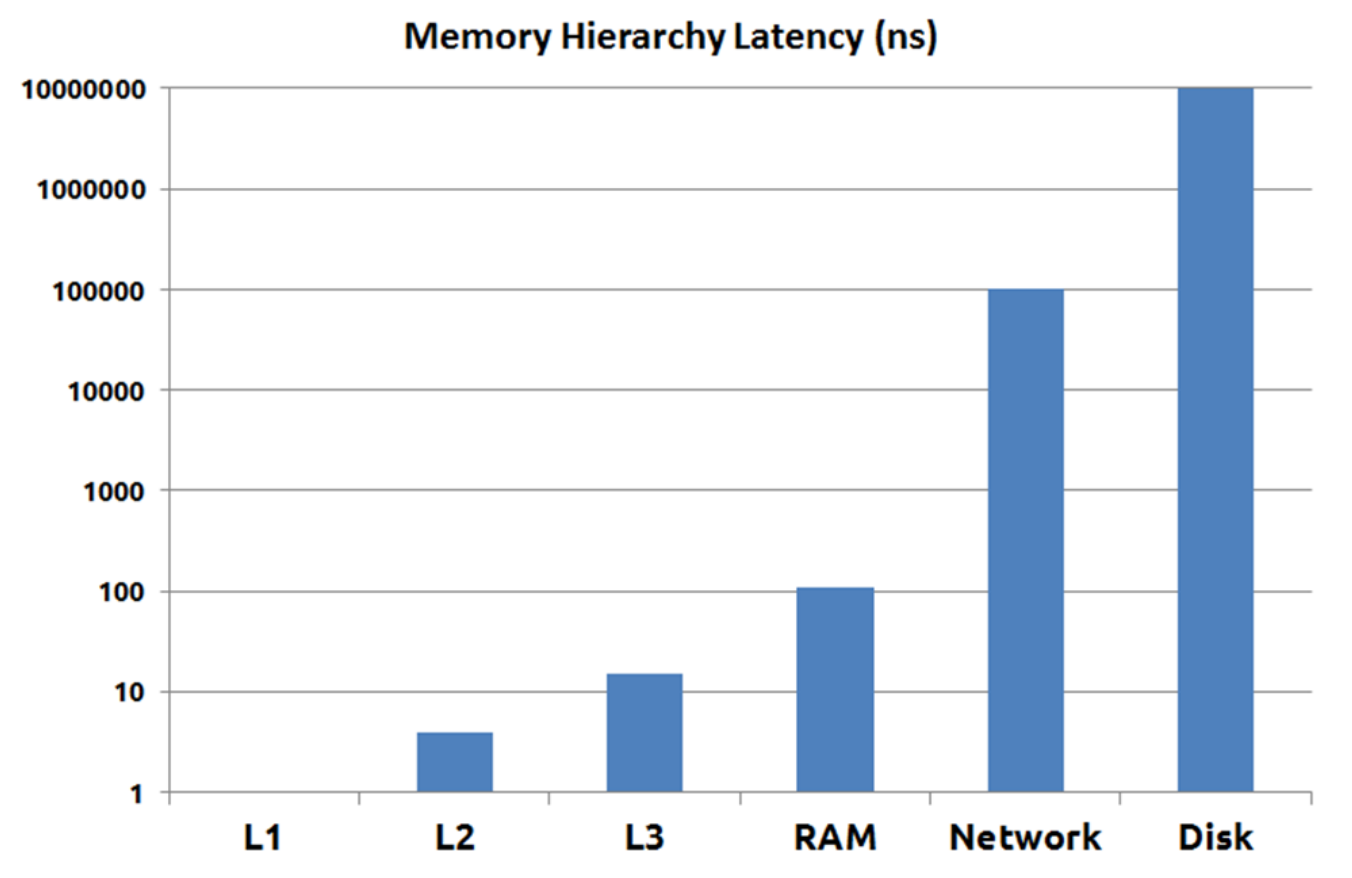

Figure 1.1: Typical memory latencies in a modern commodity machine. Gigabit Ethernet latency included for comparison. 
Because of this fact, there is a lot more to parallel rendering than initially meets the eye. Graphics may indeed by highly parallel, but its voracious appetite for memory access is actively working against its parallel efficiency on current architectures.

\subsection{Our Contribution}

This paper presents the architecture of FlexRender, a ray tracer designed for rendering huge scenes with high geometric complexity on commodity hardware. We specifically target commodity hardware because it currently has an excellent cost to performance ratio, but still typically lacks enough memory to fit large scenes entirely in RAM.

Current strategies for parallelizing a renderer across a cluster of commodity machines are limited to having each worker compute a separate "slice" of the image, but do nothing to manage the high cost associated with large scene assets.

Thus, our work describes the following core contributions:

1. A system for ray tracing which uses the pooled memory of a cluster of commodity machines to keep the entire scene in-core.

2. A method for passing ray messages between workers in the cluster with enough state to never require a reply message.

3. An extension to the stackless BVH traversal algorithm presented by Hapala et al. [8] that makes it possible to suspend traversal at one worker and resume it at another. 
4. A discussion of the concepts involved and an analysis of the resulting implementation.

In particular, we show that FlexRender can achieve speedups that are around an order of magnitude better than the traditional parallelization approach, and can naturally self-regulate the cluster of workers to keep the memory overhead due to message queuing around $1 \%$ of each worker's system memory. 


\section{Chapter 2}

\section{Background}

The FlexRender architecture builds on the four fundamental building blocks described here. First, we discuss the linearity of light (Section 2.1), which is critical to understanding why FlexRender produces a correct composite image from pieces rendered by different machines. Next, we give a general overview of ray tracing (Section 2.2) and bounding volume hierarchies (Section 2.3). Finally, we discuss Morton coding and the Z-order curve (Section 2.4) which FlexRender uses to distribute scene data amongst machines in the cluster.

\section{$2.1 \quad$ Light and Radiometry}

Radiometry, the study of propagation of electromagnetic radiation, forms the basis for many rendering algorithms. Our coverage will be brief, but we will examine some critical theory that enables the design of FlexRender. For a more complete treatment of the topic in the context of rendering algorithms, we refer the reader to Physically Based Rendering [16]. 
At its core, radiometry is based on modeling light as radiant energy and operates at the level of geometric optics. In other words, we acknowledge that light has wave-like properties and visible light occurs within a spectrum of wavelengths (roughly 400 to $750 \mathrm{~nm}$ ), but we do not mathematically model it as a wave. Rather, we model it as a particle of radiant energy. In ray tracers (discussed in depth in Section 2.2) we model the path traveled by a single particle of light with a ray.

The interesting parts of the radiometry model with respect to FlexRender are the following observations:

Light behaves linearly. The combined effect of two rays of light in a scene is the same as the sum of their independent effects on the scene.

Energy is conserved. When a ray reflects off of a surface, it can never do so with more energy than it started with.

In particular, these assumptions allow us to make the following key observations, which FlexRender explicitly exploits:

The location of computation does not matter. If the scene is distributed across many machines, it makes no difference which machine computes the effect of a ray. The sum of everyone's computations will be the same as if all the work was performed on a single machine.

Transmittance models energy conservation. If we store the amount of energy traveling along a ray (the transmittance) with the ray itself, we need not know anything about the preceding rays or state that brought this ray into existence. We can compute its contribution to the scene independently and ensure that linearity and energy conservation are both respected. 


\subsection{Ray Tracing}

Ray tracing is a method for rendering a scene from some input description. The ray casting algorithm, published by Appel in 1968 [1], proposed shooting rays out of the camera and computing their intersections with mathematically defined surfaces. One ray is shot through the center of every pixel, and the first object that ray intersects is what is seen through the camera at the pixel.

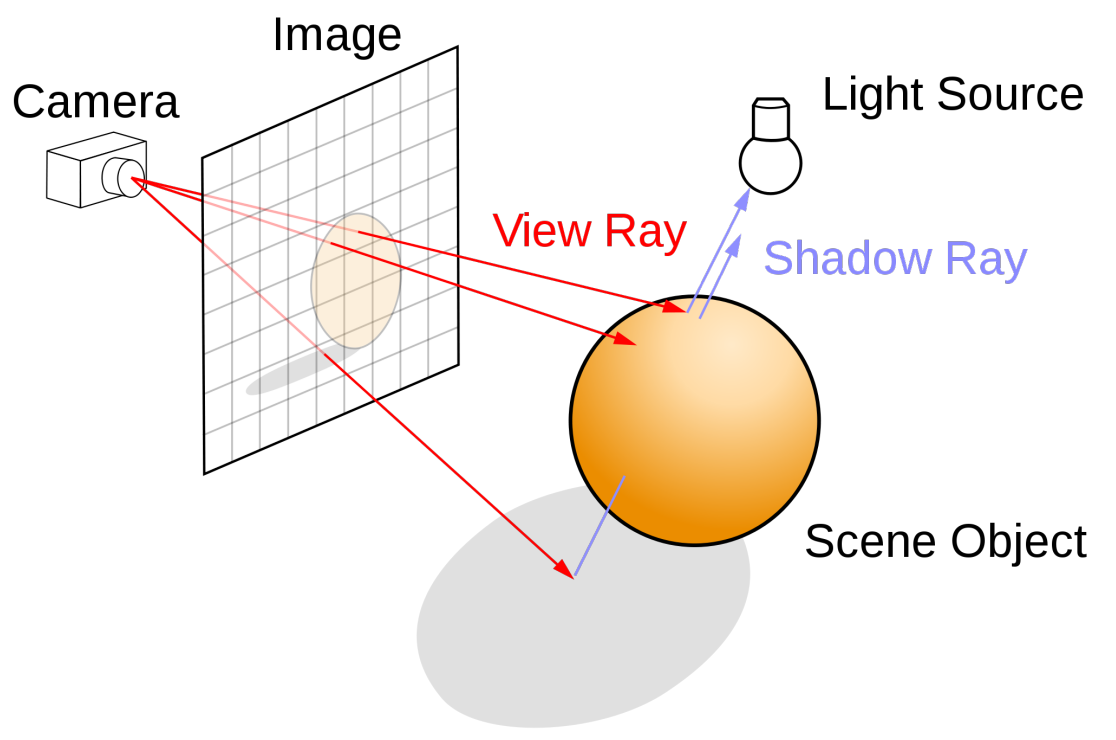

Figure 2.1: Ray tracing models light by shooting rays into the scene from the camera. Credit: "Henrik" (Creative Commons License)

In 1980, Whitted published an extension of this idea [17], which continued the process at the point of intersection. At that point a shadow ray is cast from the intersection point to the light to determine if the point is illuminated or in shadow. He also introduced the notion of tracing rays recursively, casting new rays into the scene, originating at the intersection point, whose directions are computed by reflecting or refracting them about the surface normal at the point of intersection.

Ray tracing has significant advantages over other rendering algorithms (such 


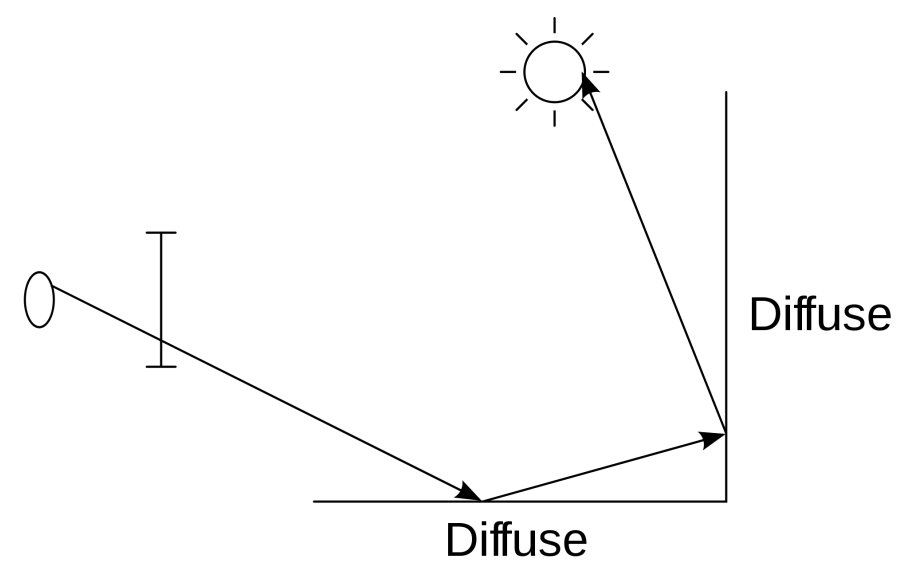

Figure 2.2: Reflection and refraction are modeled by casting new rays from intersection points. Credit: "Stannered" (Creative Commons License)

as scanline rasterization) because it more closely models the way light acts in the real world. Effects that are difficult to simulate using other methods (such as shadows and correct reflections) fall out of the the algorithm naturally, making it an attractive choice.

However, it suffers from performance problems. Without a method for reducing the number of intersection tests in a scene, each ray must be tested against every object, leading to $O(n)$ performance. Since rays are cast for each pixel in the image (or multiple rays per pixel if stratified supersampling is used for antialiasing) the number of rays scales with the resolution of the output image.

Lastly, to approximate shadows with soft edges and other subtle lighting effects, Monte Carlo integration is typically used when casting the shadow rays to the light. This means that for every intersection, $n$ sample points are computed on the surface of the light and a shadow ray is cast from the intersection point to each sample point. The results of those casts are weighted by $\frac{1}{n}$ when computing the shaded value at a point. Reflection and refraction also increase the number of rays in play. To compute global illumination (the effect of light reflecting off 
of all surfaces in the scene), the ray count increases dramatically.

Although it is common to see an exponential explosion of rays, thankfully we have algorithms for significantly reducing the number of intersection tests we need to compute per ray, which we will discuss in Section 2.3.

\subsection{Bounding Volume Hierarchies}

Bounding volume hierarchies, or BVHs, are essentially an application of binary search to 3D space. In ray tracing, the vast majority of time is spent computing intersection tests to determine whether a ray intersects a given primitive. BVHs allow us to search 3D space for a potential intersection in a binary tree fashion, pruning off large numbers of primitives with a single test. Since it is effectively binary search, it is unsurprising that it reduces intersection searching from linear time $O(n)$ to $\operatorname{logarithmic}$ time $O(\log n)$ (assuming the BVH is well formed).
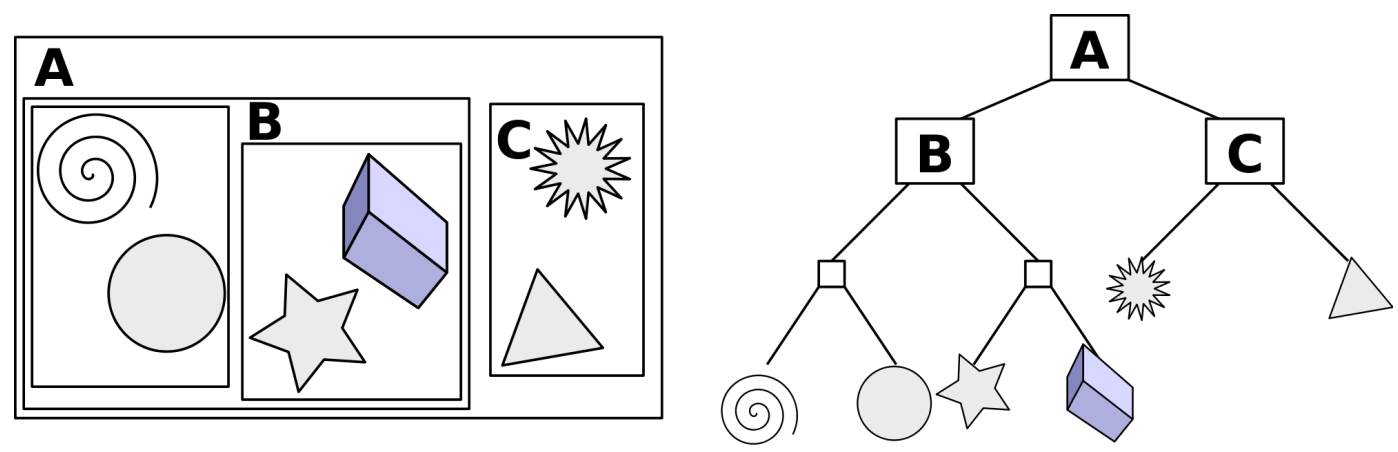

Figure 2.3: Bounding volume hierarchies partition primitives into a binary tree structure. Credit: "Schreiberx" (Creative Commons License)

BVHs are trees where each node is defined by a bounding volume, such as a box or a sphere, that describes the extents of a region of $3 \mathrm{D}$ space. All of the 
primitives in the scene that are within that region of space are child nodes in the tree. Each node has two direct children, which also define bounding volumes within that subregion of space. Nodes which have other bounding volume nodes as their children are interior nodes. Leaf nodes in the tree define a bounding volume around a single primitive.

After the tree structure is built using a typical linked-memory data structure, it is flattened into a linear array of nodes for storage efficiency and better cache performance. Flattening is done by walking the tree in a depth-first traversal. This means that the left-hand child of the node at index $n$ is located at index $n+1$ in the array of nodes. Each node stores an offset index to its right-hand child. Because the indices are relative to the array and not the array's location in memory, they are safe to pass back and forth between different machines and require no address translation.

To traverse the tree looking for intersections, we begin by testing the root node's bounding volume for an intersection. If the ray intersects the volume, we recursively test each of the node's children. Once we hit a leaf node, we perform an actual ray-primitive intersection test to determine if the ray hits the primitive.

The traversal algorithm is naturally recursive, but recursive implementations keep their state on the call stack. In FlexRender, we may need to suspend the traversal on one machine and resume it on another, so we need all of the traversal state explicitly exposed. Refactoring it as an iterative traversal explicitly exposes the state for capturing.

The iterative algorithm still requires a traversal stack of child nodes that need to be visited on the way back up the tree. Since this stack is unique for each ray, the entire stack would need to be carried along with each ray. In addition, the 
stack could potentially be large if the scene is huge (and thus, the tree is deep), so it would be ideal if we had a method for traversing the tree iteratively without the need to maintain a traversal stack.

A method for doing so is described by Hapala et al. in Efficient Stack-less BVH Traversal for Ray Tracing [8]. Their key insight is that if parent links are stored in the tree, the same traversal can be achieved using a three-state automaton describing the direction you came from when you reached the current node (i.e. from the parent, from the sibling, or from the child). They show that their traversal algorithm produces identical tree walks and never retests nodes that have already been tested.

FlexRender leverages this traversal algorithm due it its low state storage requirements. Each ray only needs to know the index of the current node it is traversing (in the array of flattened nodes) and the state of the traversal automaton. The extensions made to the algorithm to support suspending traversal and resuming it on another machine are described in Section 4.3.3.

\subsection{Morton Coding and the Z-Order Curve}

Morton coding is a method for mapping coordinates in multidimensional space to a single dimension. In particular, walking the multidimensional space with a Morton coding produces a space-filling Z-order curve.

More concretely, FlexRender needs a way to distribute a large scene to all the machines in the cluster in a spatially coherent way. If the geometry on each machine consists of a localized patch of the overall geometry, it allows us to minimize communication between the machines, and thus, only pay the network 

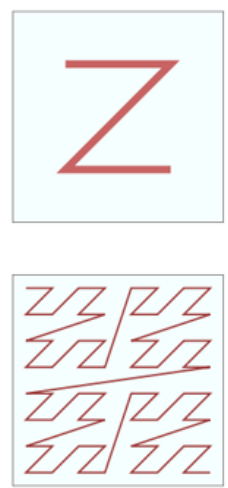
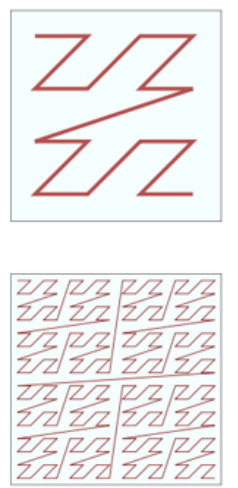

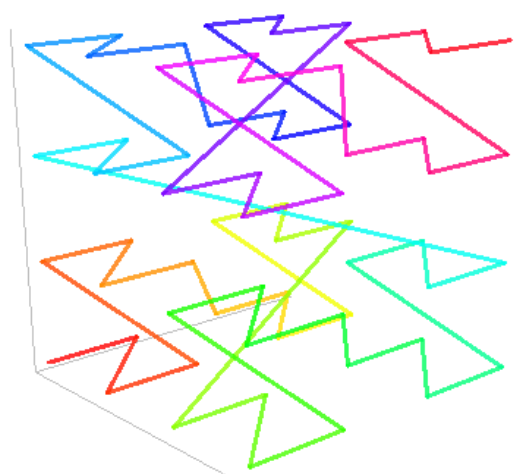

Figure 2.4: Examples of two dimensional and three dimensional Zorder curves. Credit: "David Eppstein" and "Robert Dickau" (Creative Commons License)

cost when we absolutely need to (described in depth in Section 4.3.3).

Because the Morton coding produces a spatially coherent traversal of 3D space, dividing up the 1D Morton code address space among all the machines participating in the render gives a reasonable assurance of spatial locality for the geometry sent to each machine.

The Morton coding is relatively simple to implement. For example, say that we wish to map a point $P$ in a region of $3 \mathrm{D}$ space (defined by its bounding extents min and $\max$ ) to a Morton coded 64-bit integer. Discretizing each axis evenly allows for 21 bits per axis, yielding a 63-bit address space (and one unused bit in the integer).

Computing the Morton code is simply a matter of calculating the 21-bit discretized component of $P$ along each axis, then shifting the components from each axis into the 64-bit integer one bit at a time in a round robin fashion, from the most-significant to least-significant bit.

\footnotetext{
$1 \quad / /$ Discretize each component based 21 bits per axis.

2 int factor $=0 \times 1<21$;
} 


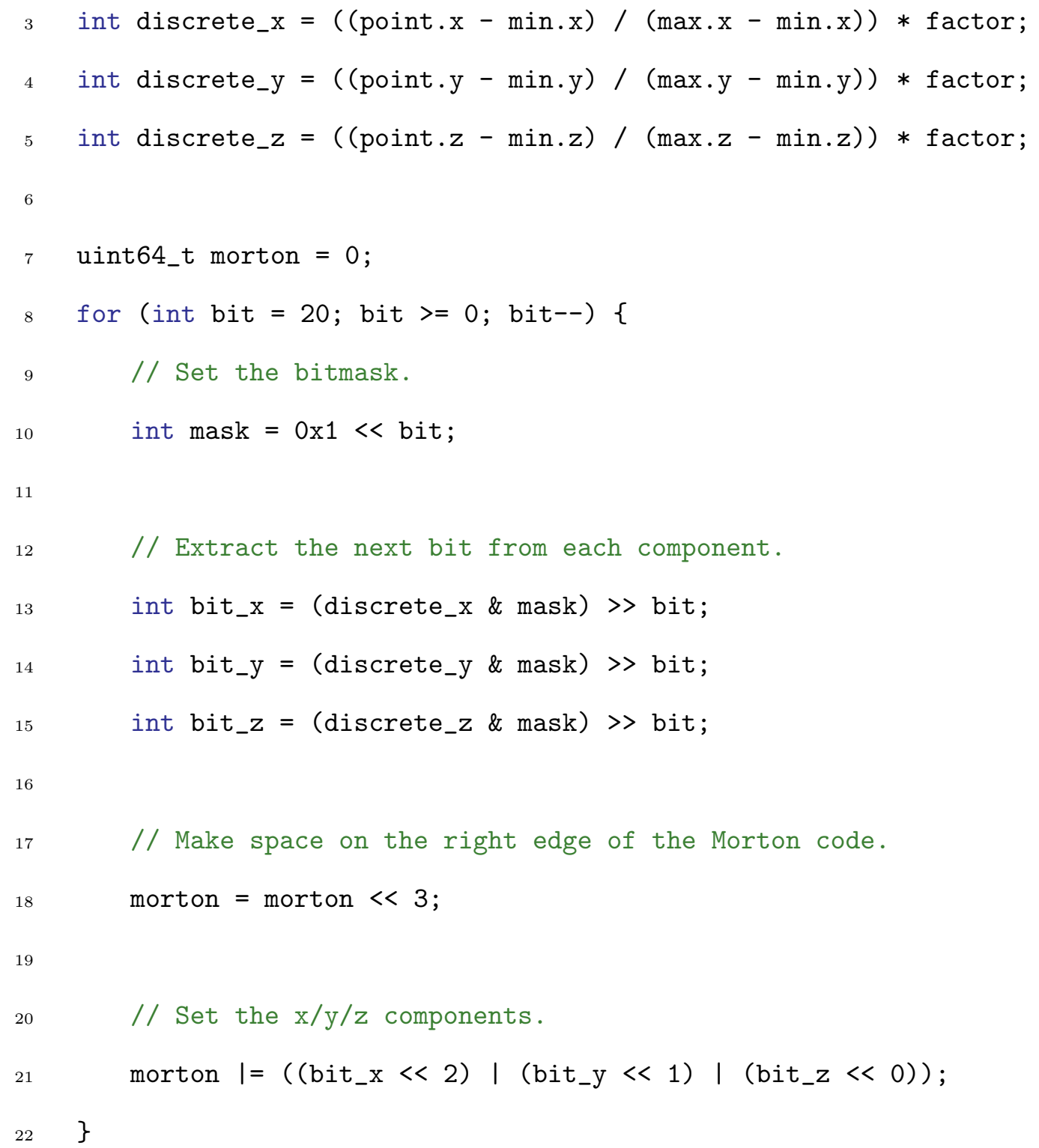

3

4 int discrete_y $=(($ point.y $-\min \cdot y) /(\max \cdot \mathrm{y}-\min \cdot \mathrm{y})) *$ factor;

5 int discrete_z $=(($ point.z $-\min . z) /(\max . z-\min . z)) *$ factor;

6

11

Listing 2.1: Example of computing the Morton code. 


\section{Chapter 3}

\section{Related Work}

Finding ways to deal with huge geometry has been a common theme in computer graphics for quite a while. Early techniques generally focused on faking high detail through clever use of textures and model swapping, since the hardware of the day simply was not capable of handling massive amounts of data.

More recently, techniques have focused around actually having the full geometry available, but acknowledging that it will not fit in main memory and providing methods for efficiently caching data that is out-of-core.

We examine these workarounds in Section 3.1, and provide a brief overview of relevant work in parallel rendering in Section 3.2. We specifically discuss the Kilauea renderer, which had similar ambitions as FlexRender but is significantly different in several ways. 


\subsection{Big Geometry Workarounds}

\subsubsection{Normal Mapping}

Normal mapping is a technique for simulating high resolution detail on a mesh during shading while using low resolution geometry. The idea of extracting detail from a high resolution mesh was first proposed by Krishnamurthy and Levoy in 1996 [11], which fit displacement maps over from non-uniform rational B-splines (NURBS).

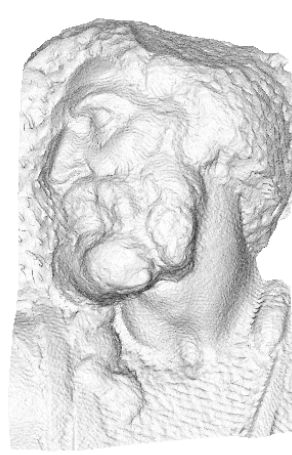

original mesh $4 \mathrm{M}$ triangles

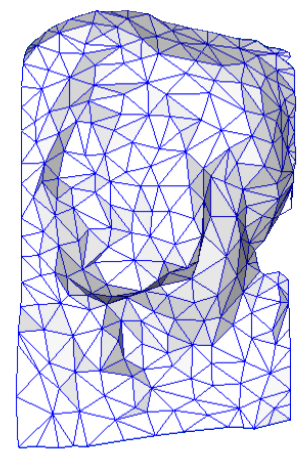

simplified mesh 500 triangles

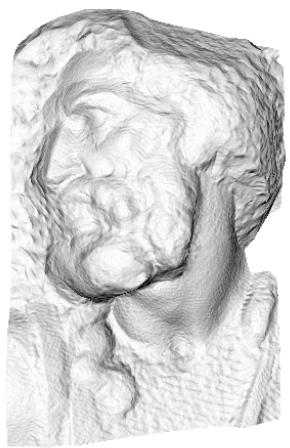

simplified mesh
and normal mapping 500 triangles

Figure 3.1: Normal mapping uses a texture to store high resolution shading normals on low resolution geometry.

Two additional papers completed the puzzle. In 1998 Cohen et al. [4] presented a method for rendering high detail at shading time by storing normals in the texture instead of using displacement maps, and Cignoni et at. [2] showed how this could be done with two meshes that were created independently.

Normal mapping is extensively used today, especially in real-time graphics applications. However, it displays visual artifacts, particularly around the edges of the mesh (where it is clearly apparent the surface is not high detail) or areas where the texture is scrunched or stretched. 


\subsubsection{Level of Detail}

Level of detail is a technique for replacing high resolution meshes with lower resolution versions when they are some specified distance away from the viewer. The idea was pioneered by James H. Clark in 1976 [3], when he noticed that there was no reason to use high resolution geometry when the resulting representation on the screen was tiny.

This technique is also still heavily used in real-time graphics applications today. However it suffers from a few problems. The first is that multiple resolutions of the mesh must be available. The second is that it is somewhat dependent on the size that the final image is viewed. A mesh may be small in screen space compared to the size of the image, but on a movie-theater sized screen it could very well still be quite large when viewed.

\subsubsection{Out-of-Core}

Recent research have acknowledged that rendering data simply will not fit into main memory any more, and have developed techniques for efficiently managing a cache of rendering data in main memory. In 2011, Tabellion et al. [10] presented a technique for dealing with huge point clouds used in global illumination. They showed that their method can process $88 \mathrm{~GB}$ of point cloud data with a cache of just $2 \mathrm{~GB}$, and can render $6.8 \mathrm{~GB}$ of point cloud data with cache hit rates of over $99 \%$.

One year earlier, Pantaleoni et at. [15] showed that their software developed for Avatar also was very efficient at dealing with out-of-core data. They used ray tracing to generate directional occlusion data, which was compressed into 
spherical harmonics. The spherical harmonics were used at render time (they did not directly ray trace the scenes during rendering). In particular, they also used hierarchical BVHs with a single "top-level" tree as FlexRender does. However, they used it for chunking the scene into manageable units of work that could be loaded and unloaded from main memory quickly.

\subsection{Parallel Rendering}

\subsubsection{Cooperative Networked Rendering}

Without a doubt, the most similar piece of research to FlexRender is Kilauea [9], a massively parallel networked renderer developed at Square USA in 2002. Similarly to our work, it uses worker machines connected on a network to keep the entire scene geometry in core. However, there are three main differences in our approach.

\section{Geometry Distribution}

Kilauea randomly distributes scene geometry to its workers, then casts intersections rays through all workers simultaneously. This may produce multiple candidate intersections. The results from each worker are gathered and a winner is picked using a standard depth test. This makes the pipeline highly sensitive to extra latency on any of the workers, because everyone is waiting for the results to run the depth test.

In contrast, FlexRender attempts to keep geometry spatially local among its worker machines. This allows us to build a hierarchical spatial acceleration structure over the workers and reduce the number of network hops we need to make to establish the correct intersection. 


\section{Shading Secondary Rays}

When Kilauea decides to compute the shading value of a bounced ray, it suspends execution of the shader and keeps it around until the bounced ray comes back through the system. Similarly to FlexRender, they keep a priority queue for rays to process those most likely to terminate first.

FlexRender maintains the source pixel and transmittance of each ray as state, so the suspension of shaders (and associated overhead) is unnecessary. Each worker has its own image buffer, which is composited into the final image at the end, respecting linearity.

\section{Adaptability to Heterogeneous Architectures}

Because Kilauea needs the results from every worker before performing the depth test, it is extremely latency sensitive. This encourages homogeneity among workers, and makes adding new workers into the cluster with vastly different machine architectures (such as GPGPU) or performance characteristics problematic.

In contrast, workers in a FlexRender cluster are never waiting on the results from another worker. Thus, new machines can be dropped into the cluster without regard for how they will effect the latency of the entire system. For example, a GPGPU worker might batch up requests and outsource them to the GPU only when it can effectively amortize the cost of the memory bus transfer.

\subsubsection{General Purpose GPU Rendering}

As interest in General Purpose GPU, or GPGPU, computing has increased over the last several years, many have decided to port ray tracers to the archi- 
tecture due to the problem's "embarrassingly parallel" nature. Although lots of simple implementations are floating around, only relatively recently have researchers started tackling the challenging problems imposed by writing a serious ray tracer on a GPGPU architecture device. It may be deceptively straightforward to get a simple implementation up and running, but the complexity is in the features and details required by a robust renderer.

Probably the most impressive recent work was done by Garanzha et al. [6], which presented a method for ray tracing large scenes that are out-of-core on the GPU. Parts of their architecture are similar to FlexRender on a single-device scale, specifically their use of multi-level BVHs for data that is paged into memory and their use of ray queues to handle rays cast from shaders. Their global ray queue, however, is fairly memory intensive, because it can potentially store up to 32 million rays.

Another area of intense research has been spatial data structure construction on the GPU. Although they usually produce data structures of slightly lower quality than their CPU counterparts, these GPU algorithms go to great lengths to produce "high-enough" quality structures with dramatic speedups. Garanzha et al. [7] present a method for constructing BVHs on the GPU and Wu et al. [18] present a method for constructing KD-trees on the GPU. Both are guided by the surface area heuristic.

\subsection{MapReduce}

Outside of the graphics world there has been great excitement surrounding the MapReduce programming paradigm presented by Dean and Ghemawat in 2004

[5]. For smaller scenes whose geometry and acceleration structure data would fit 
entirely in-core on a single worker, this may be an interesting approach worth exploring.

Northam and Smits [14] presented a potential MapReduce implementation of a ray tracer, but noted that sending large amounts of scene data to the workers significantly slowed down scene processing. Their solution was to break the scene into small chunks and resolve the winning intersection test in the reduce step. While this works just fine, it also does a lot of unnecessary work, because many non-winning intersections are computed that would have been pruned in a typical BVH traversal. 


\section{Chapter 4}

\section{FlexRender Architecture}

In this chapter, we describe the network architecture and roles of the involved machines in Section 4.1.1. We discuss the structure of ray messages passed between workers in Section 4.1.2 and briefly cover the design of the graphics machinery shared between FlexRender and the baseline implementation in Section 4.1.3. We detail the process of preparing the cluster for rendering in Section 4.2.1 and Section 4.2.2.

At its core, workers in the cluster are just ray processors. We discuss how they manage ray messages in Section 4.3.1, how they generate new work with stable memory usage in Section 4.3.2, how we decide when and where to send rays over the network in Section 4.3.3, and how shading is computed when lights and occluders may exist anywhere in the cluster in Section 4.3.4.

Finally, we wrap up our discussion with how we monitor the progress of the render in Section 4.4.1 and how we composite the final image from its components in Section 4.4.2. 


\subsection{Organization and Design}

\subsubsection{Workers and the Renderer}

There are two potential roles a machine can play during the rendering process.

Worker These machines receive a chunk of the scene and act as ray processors to compute intersections and shading values. They produce an image that is a component of the final render. There may be an arbitrary number of them participating in a render.

Renderer This machine reads in the scene data and distributes it to the workers.

Once rendering begins it monitors the status of the each worker and halts any potential runaway situations (see Section 4.3.2). When the renderer decides the process is complete, it requests the image components from each worker and merges them into the final image. There is only a single renderer in any given cluster and it is the machine the user directly interacts with.

From a network perspective, the architecture is simply client/server connected in a star topology. Each worker exposes a server which receives and processes messages, and holds client connections open to every other worker for passing messages around the cluster. The renderer also holds a client connection to every worker for sending configuration data, preparing the cluster for rendering (described in Section 4.2.1), and monitoring render progress (described in Section 4.4.1).

It is important to note that there is currently no fault tolerance built into FlexRender. This decision was entirely due to limited resources and time con- 
straints; there is no architectural reason that the system could not be made more robust and fault tolerant with the appropriate application of distributed systems concepts. To that end, we refer the reader to the distributed systems literature.

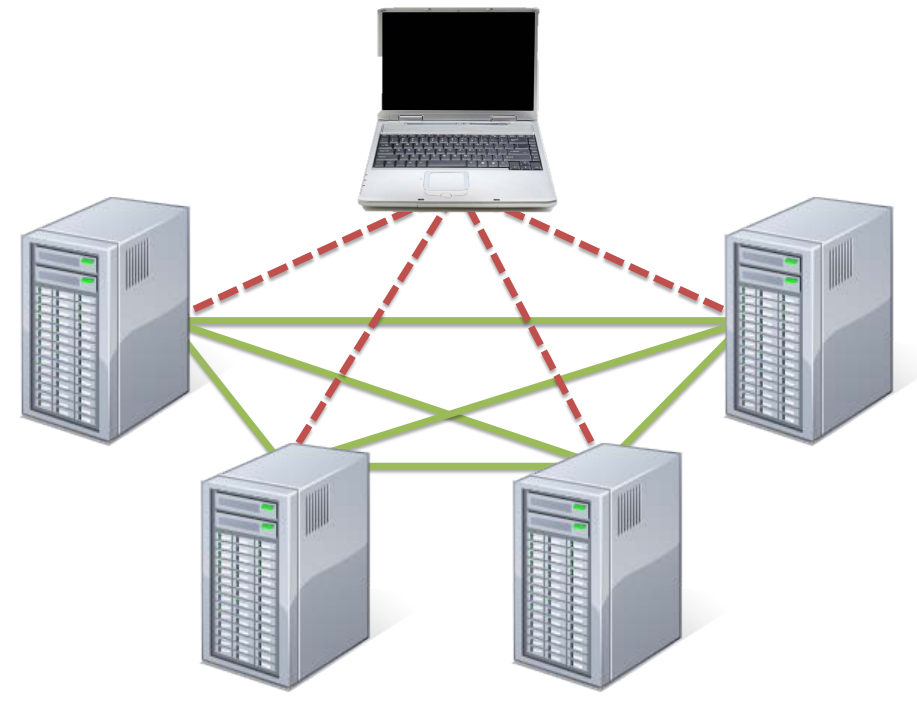

Figure 4.1: Workers maintain connections with all other workers in the cluster. The renderer does the same.

\subsubsection{Fat Rays}

The currency of computation and core message type in FlexRender is the fat ray. They are so named because they carry additional state information along with their geometric definition of an origin and a direction. Their counterparts, slim rays, consist of only the geometric components.

Specifically, a fat ray contains the following data:

- The type of ray this is. Described in Section 4.3.

- The source pixel that this ray contributes to. 
- The bounce count, or number of times this ray has reflected off a surface (to prevent infinite loops).

- The origin and direction of the ray.

- The ray's transmittance, or the amount that it contributes to the source pixel.

- The emission from a light source carried along the ray (if any). Described in Section 4.3.4.

- The target intersection point of the ray, if any. Described in Section 4.3.4.

- The traversal state of the top-level BVH. Described in Section 4.3.3.

- The hit record, which contains the worker, mesh, and $t$ value of the nearest intersection.

- The current worker this ray should be sent to over the network.

- The number of workers touched by the ray so far. Not necessary for rendering. Only used for analysis.

- A next pointer for locally queuing rays as described in Section 4.3.1. Obviously not valid over the network.

In total, the size of a fat ray is 128 bytes.

\subsubsection{General Types}

The core graphics machinery in FlexRender is fairly straightforward. A scene consists of a collection of meshes, which are stored as indexed face sets of vertices 
(positions with normals and texture coordinates) and faces.

Each mesh is a assigned a material, which is responsible for drawing the mesh. A material consists of a shader and potentially a set of bindings from textures to names in the shader.

A shader is a piece of code that is run to compute the lighting on a surface at a particular point.

A texture is a either a 2D array of pixels (for image textures) or a snippet of code (for procedural textures) that defines the value of something across a surface. They are most commonly used for providing colored detail across the face of a triangle.

All of the code used in graphics computation is shared between both our baseline implementation and FlexRender. This ensures fair comparisons when we analyze our results in Section 5.

\subsubsection{Overview}

In general, rendering with FlexRender proceeds in the following way:

1. The renderer reads in the scene data and defines the maximum bounding extents of the entire scene. As data is read in, it is distributed to the workers using the Morton coding.

2. Once the scene is distributed, workers build BVHs in parallel for their respective "chunk" of the scene. They share their maximum bounding extents with the renderer.

3. The renderer constructs a "top-level" BVH from the worker bounds and 
shares this BVH with all the workers.

4. Each worker creates its own image buffer. This is where any shading data will be written if it occurs on this worker.

5. Each worker begins casting primary rays from the camera and tests for intersections (to find the nearest geometry that the ray hits).

- During intersection testing, rays may be forwarded over the network to another worker if they travel through the bounding extents of that worker.

- Once the nearest intersection is found illumination ray messages are created and sent to workers that have lights. These workers send light rays back towards the point of intersection to test for occlusion.

- If the light rays reach the intersection, the point is shaded because it is in direct view of the light.

6. Once all rays have been accounted for, workers send their image buffers back to the renderer, which composites them all together into a final image.

In the next few sections, we will examine each part of this process in greater detail.

\subsection{Render Preparation}

Render preparation consists of ensuring that all the workers agree on the basic rendering parameters, distributing the scene assets to each worker, and building the distributed bounding volume hierarchy. We discuss configuration 
and distribution in Section 4.2.1 and parallel construction of the BVH in Section 4.2.2.

\subsubsection{Configuration and Asset Distribution}

The configuration consists of basic information about how the scene is to be rendered, such as the image dimensions, the workers participating in the render, etc. In addition, the configuration specifies the minimum and maximum bounding extents of the scene. This is used in the asset distribution step for driving the Morton coding discretization along each axis.

Once the renderer has read in the configuration, it opens client connections to each worker and sends the configuration data to them. Similarly, each worker

opens a client connection to every other worker. These renderer/worker and worker/worker connections remain open for the duration of the render.

The renderer then divides up the 63-bit Morton code address space evenly by the number of workers participating and assigns a region of it to each one.

Finally, the renderer begins reading in and parsing the scene data. As each mesh is loaded, the renderer takes the following actions:

1. Computes the centroid of the mesh by averaging its vertices.

2. Computes the Morton code of the mesh centroid. This determines which worker the mesh will be sent to.

3. Ensures that any asset dependencies (such as materials, shaders, and textures) for this mesh have been sent to the designated worker.

4. Sends the mesh data to the designated worker. 
5. Deletes the mesh data from its own memory.

Although the current implementation reads scene data in at the renderer and distributes it over the network, there is no inherent reason why it needs to do so. For example, if all the workers have access to the scene data over network storage, the renderer could simply tell each worker the range of the Morton code address space it is responsible for and let them load the scene data themselves, carefully throwing out any geometry that is not within their Morton bounds.

\subsubsection{Parallel Construction of Spatial Acceleration Struc- tures}

To accelerate intersection testing against each mesh, each worker first constructs a BVH for every mesh it has. These BVHs are tied directly to the mesh

they represent. While building each BVH, the worker stores a list of the bounding extents of each mesh.

Next, each worker builds a root BVH for the entire worker, which uses the mesh extents for its leaf nodes. When testing for intersections locally, a worker first tests against this root BVH to determine candidate meshes, then traverses each mesh's individual BVH to compute absolute intersections. After construction of this root BVH, the root node's bounding extents will describe the spatial extent of all geometry located on that worker.

Once construction of all the local BVHs is complete, each worker sends its total bounding extents to the renderer. Once the renderer has the bounding extents of each worker, it constructs a final "top-level" BVH of the workers. The renderer then distributes this top-level BVH to all the workers, so that everyone 
participating has an identical copy of it. This is a very quick and lightweight process, since it is only dependent on the number of workers participating in the render.

This top-level BVH will be used to direct where we pass rays over the network in Section 4.3.3.

\subsection{Ray Processing}

At their core, workers are essentially just multithreaded ray processors. Once rendering begins, they continually pull rays out of the ray queue (discussed in Section 4.3.1), schedule them onto the thread pool, and process them when the thread is run. This processing step consists of testing for intersection, potentially forwarding the ray to another worker (Section 4.3.3), or computing shading values if the ray terminates (Section 4.3.5).

As mentioned in Section 4.1.2, fat rays have an associated type. There are three different types of rays in FlexRender:

- Intersection rays are rays whose sole purpose is to identify a point in space at which we would like to compute shading.

- Illumination ray messages are essentially copies of intersection rays that have terminated. They are sent to workers who have emissive geometry (described in Section 4.3.4).

- Light rays are Monte Carlo samples that contribute direct illumination to a point that a worker is shading (described in Section 4.3.4).

An important point to note about these rays is the sequence of their lifetimes. 


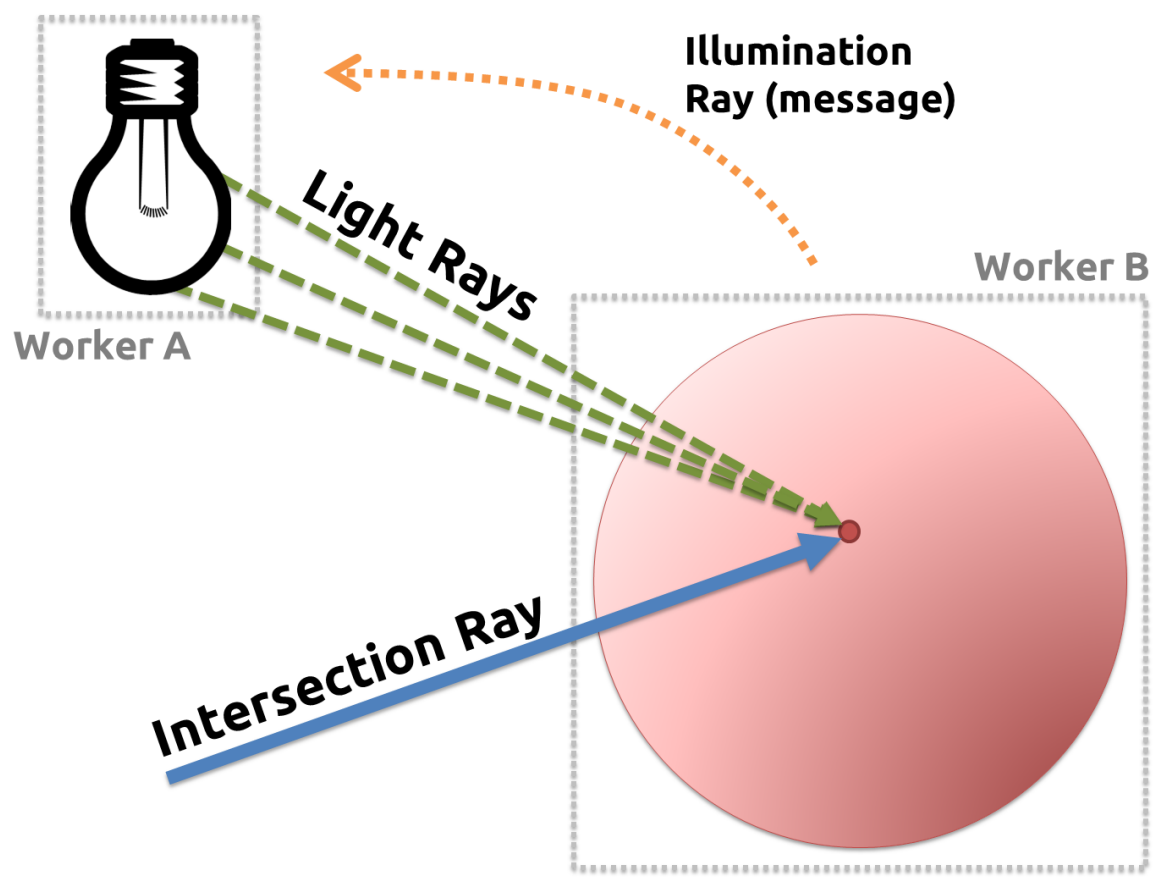

Figure 4.2: The three different ray types and their interactions.

Their purposes will become more clear in the following sections, but the order is as follows:

1. An intersection ray is cast into the scene.

2. When that intersection ray terminates at a point in space, it dies and spawns illumination ray messages.

3. When those illumination ray messages reach their destination, they die and spawn light rays.

4. When those light rays terminate at a point in space, a shading value is computed.

From this sequence of lifetimes, it should be apparent that a single intersection ray can spawn many additional rays. It should also be apparent that light rays are the most likely to die without generating more rays. 


\subsubsection{Ray Queues}

Each worker has a ray queue, with the typical push and pop operations for adding and removing rays from the queue. This queue internally is implemented as three separate queues, where rays are separated by type. It also contains information about the scene camera, for generating new primary rays.

When a new ray arrives at a worker over the network, it is immediately pushed into the queue. Internally, it is pushed into the queue matching its type (intersection, illumination, or light).

When the worker pops a ray from the queue, we pull rays from the internal queues in the following order:

1. The light queue, since these are least likely to generate new rays.

2. If the light queue is empty, we pop from the illumination queue, since these will generate a limited number of new rays.

3. If the illumination queue is empty, we pop from the intersection queue, since these can generate the most new rays.

4. If all of the internal queues are empty, we use the camera to cast new primary rays into the scene (described in 4.3.2). This effectively generates new work.

Organizing the processing order of rays as a priority queue based on ray type is an essential step in minimizing the exponential explosion of work than can occur if too many primary rays enter the system at a time. This helps reduce memory usage required for queuing messages because new work is not generated until the system is ready to handle it. 


\subsubsection{Primary Ray Casting}

The workers in the cluster are each responsible for casting a portion of the primary rays in the scene. The reasoning behind this is to give the cluster the ability to regulate itself.

Consider the case where a worker has not received any work recently from other workers in the cluster. For whatever reason (intersection tests, shading, etc.) the other workers are too busy dealing with their own queues to send any work in the direction of our lonely, rayless worker. By giving the worker control over primary ray casting for a portion of the scene, we give workers the ability to generate work when they have nothing to do.

However, to quote Uncle Ben, "with great power comes great responsibility". Consider the case where a worker contains mostly background geometry. It will be receiving work from others infrequently because its size in screen space is small, but yet it is in charge of casting primary rays for a much larger slice of the image. In this case the worker may go on an unfettered spree of primary ray casting, causing lots of grief for his fellow workers since he is essentially generating work for others and little for himself.

To prevent this "runaway" case from overburdening the ray queues, we require that workers report statistics about their progress to the renderer every so often (10 times per second in the current implementation). If the renderer notices that a worker is getting significantly further ahead of the others in primary ray casting, we temporarily disable primary ray casting on that worker until the others catch up.

Because the other workers will not generate primary rays as long as they still have other rays in their queues to process, the priority queuing, shared 
ray casting responsibilities, and temporary pausing of primary ray generation at runaway workers provides a simple means of self-regulating the cluster that works remarkably well and minimizing the memory overhead for message queuing. We will examine the overhead in detail in Chapter 5.

\subsubsection{Distributed BVH Traversal}

To understand how distributed traversal of the top-level BVH works, consider the following example traversal of this top-level tree with 5 workers (letters A through E) participating in the render.

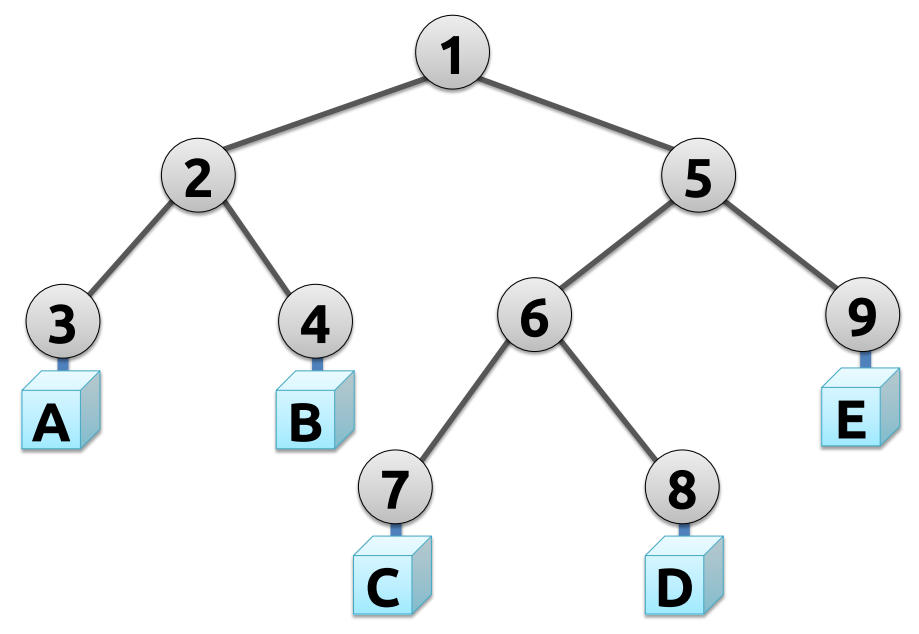

Figure 4.3: Example top-level BVH with 5 workers.

The worker who generates the ray begins traversing the tree by checking the ray against the bounds of the left-hand child. If the ray fails the test, we move to check its sibling, right-hand child. 


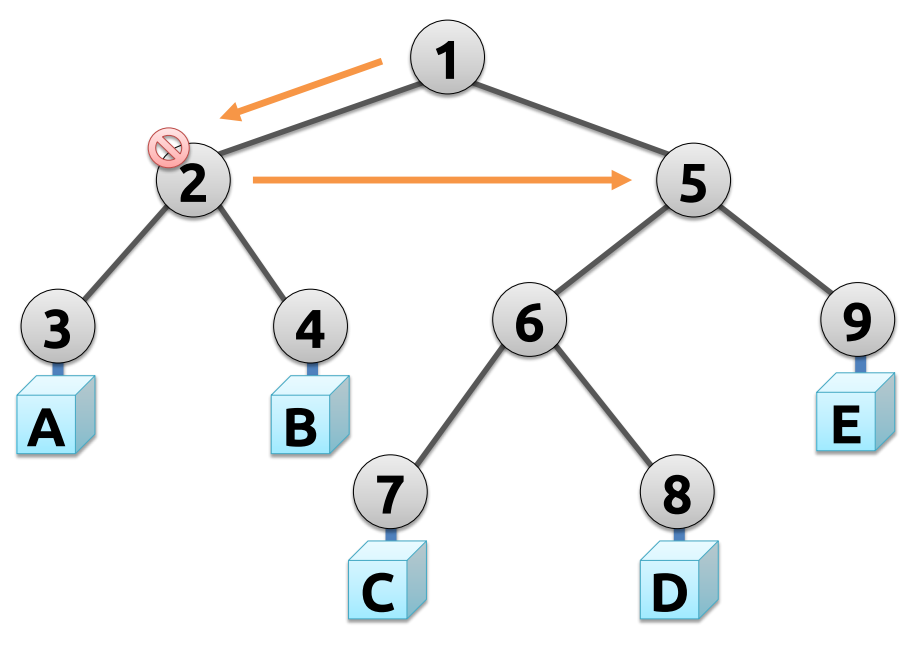

Figure 4.4: The ray fails its first test and moves to the sibling node.

This test passes, so we continue down the tree to that node's left-hand child. Let us assume this test also passes.

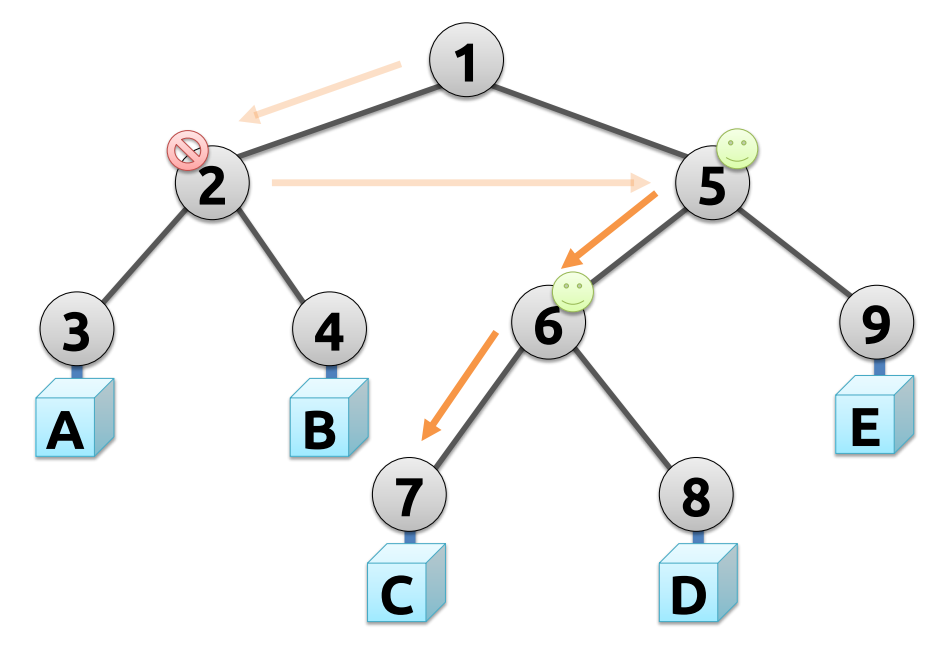

Figure 4.5: Traversal down the left-hand nodes continues as long as bounding tests pass.

We test our first leaf node, and it passes. This indicates we must test the 
geometry on the worker itself. This is where our primary modification to the Hapala et al [8] algorithm occurs. Rather than continuing to traverse the tree, we immediately jump out of the function the return the traversal state. The traversal state consists of the index of the current node, and the automaton defining where we came from ( 7 and from the parent respectively). We store this traversal state in the ray and pass it off to worker C.

When worker $\mathrm{C}$ unpacks the ray and begins to process it, it notices that the state of the traversal is not at the root of the tree, which means this is a ray whose traversal was suspended. Because a suspended ray would never be sent to a node for any other purpose than an intersection test, worker $\mathrm{C}$ immediately tests for intersections against its geometry. For the purpose of this example, let's say it hits nothing.

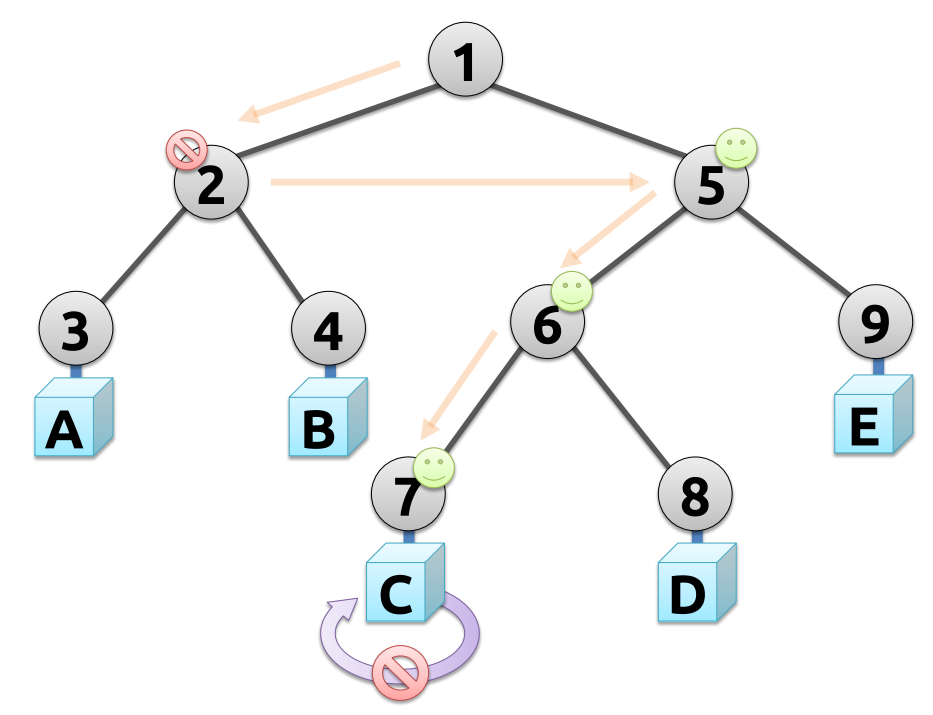

Figure 4.6: The ray is sent to worker $\mathrm{C}$ and tested against local geometry for intersections.

The traversal state is then reinstated (node 7, from the parent) and worker $\mathrm{C}$ jumps immediately to where it left off, testing the bounds of C's sibling, worker 
D. It hits the bounds, so again, we pack up the traversal state (now node index 8 and from the sibling) and ship off the ray to worker D.

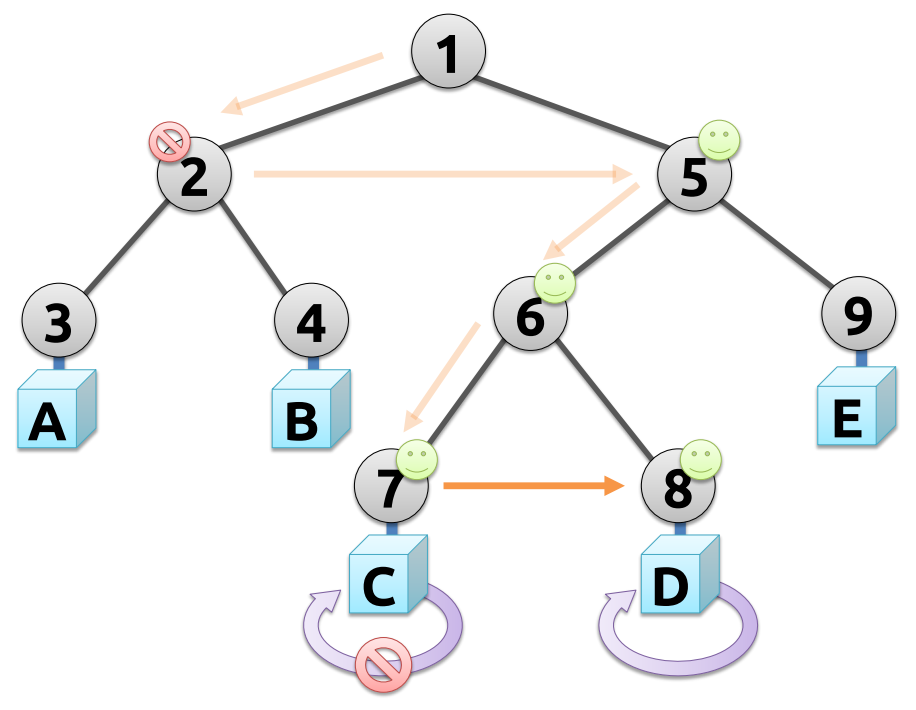

Figure 4.7: The ray is sent to worker $\mathrm{D}$ and again tested against local geometry for intersections.

Worker D goes through the same process. Since the ray is suspended, it checks its local geometry for intersections. Let's say it hits a mesh 42 at distance of 10 units along the ray. This forms a hit record, which is stored inside the ray (worker D, mesh 42, 10 units).

Again we reinstate the traversal state (node 8, from the sibling) and traverse back up to the parent (node 6). Since our state entering node 6 is from the child, we move next to node 9 , another leaf node, and test its bounds against the ray. 


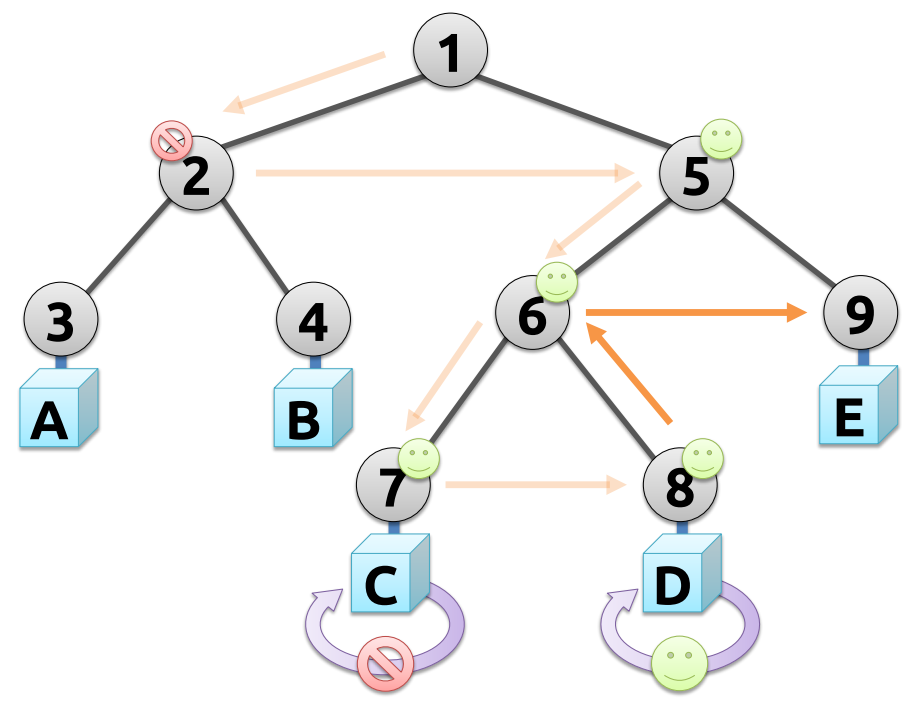

Figure 4.8: With successful hit record in hand, traversal continues back up the tree.

Let's say this also passes. However, the ray intersects the bounds at a distance of 20 units along the ray. The closest an intersection on worker E could possibly occur is further away, so there is no point to sending the ray to worker E. Thus, we do not send the ray to worker E and continue to traverse up the tree until we reach the root again. 


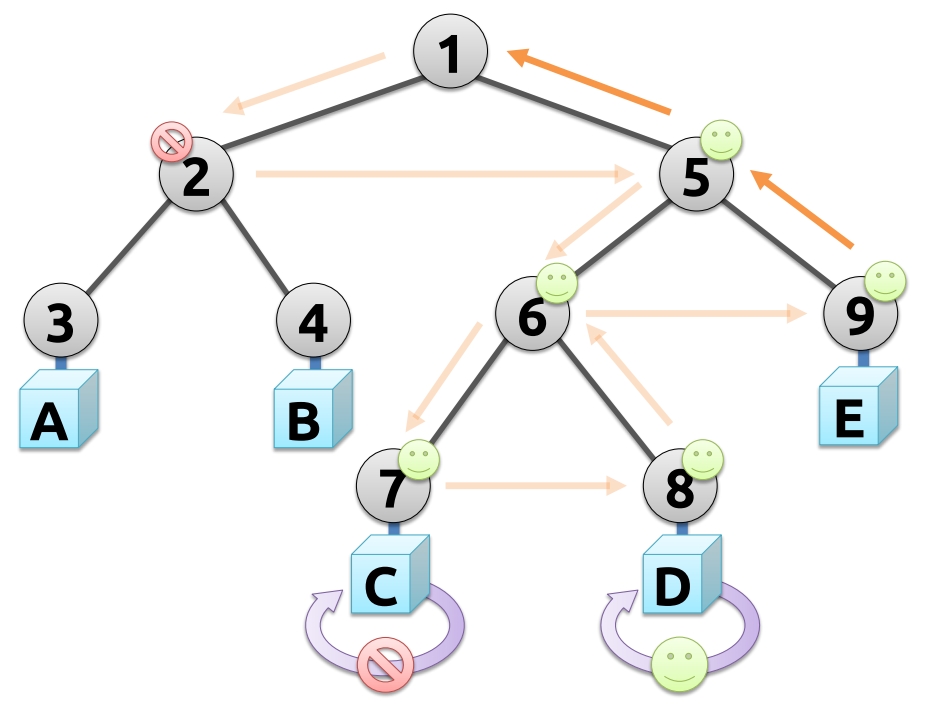

Figure 4.9: Worker E's bounding test passed, but was further away than the hit record. We arrive at the root and traversal is complete.

Once we reach the root, we know the top-level traversal is complete. At this point we only need to consult the ray's hit record to determine which mesh on which worker yields the closest intersection.

In the best case, a ray is generated on, intersects only with, and is shaded on a single worker. These rays never need to touch the network. In the worse case, the ray potentially intersects with all nodes, so it must touch every node in the tree before being sure of its intersection point.

In addition, there are two interesting corner cases to consider.

- If the ray is generated on a node that it does not intersect until later in the traversal, it consumes one additional network hop at the very beginning of the traversal.

- If the ray completes its traversal on a worker different from that which had the closest intersection, it consumes one additional network hop at the end 
of the traversal to put the ray on the correct node for shading.

Thus, the worst case scenario actually touches $n+2$ workers, where $n$ is the number of workers in the cluster.

In Chapter 6, we discuss possible optimizations that may eliminate both of these corner cases. Additionally in Chapter 5, we show that in practice, the number of workers that each ray touches is less than or equal to the predicted $O(\log n)$ expansion of the binary tree for the majority of rays in the scene.

\subsubsection{Illumination}

There is no special "light" type in FlexRender, rather only meshes that are emissive, which is a property set by the assigned material. Meshes that are emissive are known to inject light into the scene through a special function in their shader which is called to compute that emission. During scene loading, the renderer maintains a list of all workers that have received at least one emissive mesh. This list is synchronized with all workers right before rendering begins.

Once a point has been identified for shading with intersection testing, we need to determine the visibility of that point with respect to the light sources in the scene. In a traditional ray tracer, you simply cast rays from the point of intersection to samples on the surface of the light to determine visibility. However in FlexRender, from the perspective of the worker doing the shading, we have no idea where all the of the lights in the scene are, or more importantly, if there is any geometry occluding the light and casting the intersection in shadow.

To solve this problem, we simply trace the light rays in the opposite direction. Rather than originating from the intersection point and heading in the direction 
of the light, we originate the rays at the light and cast them in the direction of the intersection point. To support area lights through Monte Carlo integration, it is simply a matter of creating multiple light rays with origins sampled across the surface of the light.

If the light ray arrives back at the original intersection point after being traced through the cluster, we know that its path from the light was unoccluded. This leverages the same efficient method for distributed ray tracing that we already developed for testing intersections.

Specifically, casting light rays towards an intersection point consists of the following operations:

1. At the worker where an intersection was found, copy the ray data into an illumination ray message with the target set to the point of intersection.

2. Send this illumination ray message to all workers known to have emissive meshes.

3. When a worker receives an illumination ray message, generate sample points across the surface of all emissive meshes. Set these sample points as the origins for new light rays.

4. Set the directions of all the light rays such that they are pointed in the direction of the target (the original point of intersection).

5. Push each light ray into the ray queue and let the cluster process them as usual. 


\subsubsection{Shading}

When a light ray arrives at a worker, we simply check that its point of intersection is within some epsilon of the target. If it is, we consider the light sample visible, look up the material, shader, and textures for the mesh, and run the shader. The shader is responsible for writing its computed values into the worker's image buffer.

The implementation of shaders in FlexRender is through extensions to the Lua [12] programming language, using the LuaJIT [13] implementation for speed. A shader may do any (or none) of the following:

1. Sample textures based on the name bindings assigned in the material definition.

2. Compute a light value based on some implementation of a mathematical shading model (such as the Phong model) and local information at the point being shaded (such as the interpolated surface normal and texture coordinates).

3. Accumulate computed light values into the primary RGB buffers, or any auxiliary named buffer.

4. Cast additional rays into the scene. 


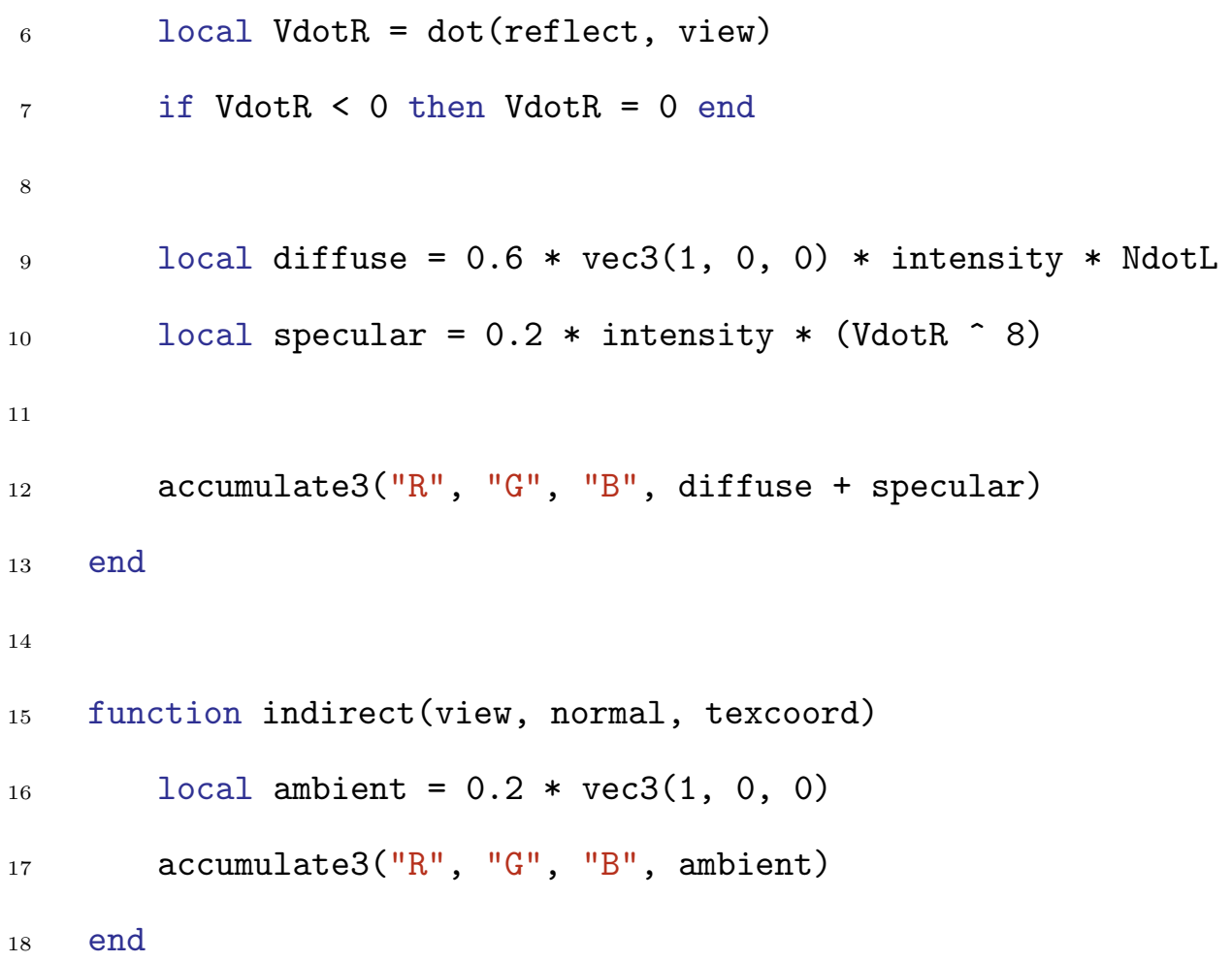

\section{Listing 4.1: Example of a simple Phong shader.}

When new rays are cast into the scene from a shader, the results of that trace are not immediately available. Instead, the trace pushes the new rays into the queue for processing and the traversal and shading systems ensure that the result of secondary and $n$-ary traced rays will be included in the final image.

In order for linearity and energy conservation to be respected, certain values are inherited from the parent ray. In particular, the source pixel is inherited (so the ray contributes to the correct pixel in the final image) and the desired transmittance along the new ray is multiplied with the transmittance of the parent ray (to ensure energy conservation is preserved).

Casting rays from the shader can be used to implement several common visual effects: 
- Alpha masking can be achieved by casting a new ray in the same direction with the full transmittance of the parent ray.

- Reflection can be achieved by casting a new ray in the reflected direction with some fraction of the transmittance based on the reflectivity of the surface.

- Refraction can be achieved by casting a new ray in the refracted direction with some fraction of the transmittance based on the index of refraction and opacity of the surface.

- Monte Carlo global illumination can be achieved by casting rays in random directions by sampling the hemisphere above the surface, with fractional transmittances based on the number of samples.

\subsection{Render Completion}

In a traditional recursive ray tracer, determining when the render is complete is a simple task. Once the last primary ray pops its final stack frame the render is over. In FlexRender, however, no one worker (or the renderer, for that matter) knows where the "last ray" is. To determine when a render is complete, the workers report rendering statistics to the renderer at regular intervals, which are used for deciding when the render is finished. This decision metric is discussed in Section 4.4.1.

Once the render is complete, the renderer requests the individual image buffers from each worker and reassembles them into the final output image. This process is discussed in Section 4.4.2. 


\subsubsection{Statistics and Monitoring}

For monitoring the progress of the render, the workers report general statistics about their activities to the renderer at regular intervals (in our current implementation, 10 times per second). In particular, the following four statistics are useful for determining if the render has finished:

Primary Ray Casting Progress The amount of the worker's primary rays that have been cast into the scene.

Rays Produced The number of rays created at the worker during this measurement interval. This includes new intersection rays cast from the camera or a shader, illumination ray messages created by terminating intersection rays, or light rays created by processing illumination ray messages.

Rays Killed The number of rays finalized at the worker during this measurement interval. This includes intersection rays that terminated or did not hit anything, illumination ray messages that were destroyed after spawning light rays, or light rays whose final shading value was computed.

Rays Queued The number of rays currently in this worker's ray queue.

In particular, if any worker has not finished casting its primary rays, we know for certain the render is not complete. Secondly, if we observe that no rays are produced, killed, or queued at any workers for some number of consecutive measurement intervals, it is reasonable to assume that the render has concluded.

Our current implementation (which reports statistics 10 times per second) waits until it sees 10 consecutive intervals of "uninteresting" activity on the workers before declaring the render complete. We find this achieves a nice balance 
between wanting to end the render as soon as it is legitimately finished and risking concluding it too early.

\subsubsection{Image Synthesis}

Once the render has been deemed "complete", the renderer requests the image buffers from each worker. Because all rendering was computed by respecting linearity, computing a pixel in the final output image is just a matter of summing corresponding pixels in the worker buffers.

This can yield some interesting intermediate images. Each worker's buffer represents the light in the final image that interacts in some way with the geometry present on that worker.

For direct light, this shows up as shaded samples where geometry was present and black areas where it was not. For other effects such as reflections and global illumination, it appears that the worker had access to the whole scene's geometry all along, but this is just an optical illusion. Since the rays carry with them the source pixel they contribute to (and this value is inherited as new rays are spawned from the primary ray), a worker can end up contributing to any pixel in the image as long as the light interacted in some way with the geometry it controlled. 


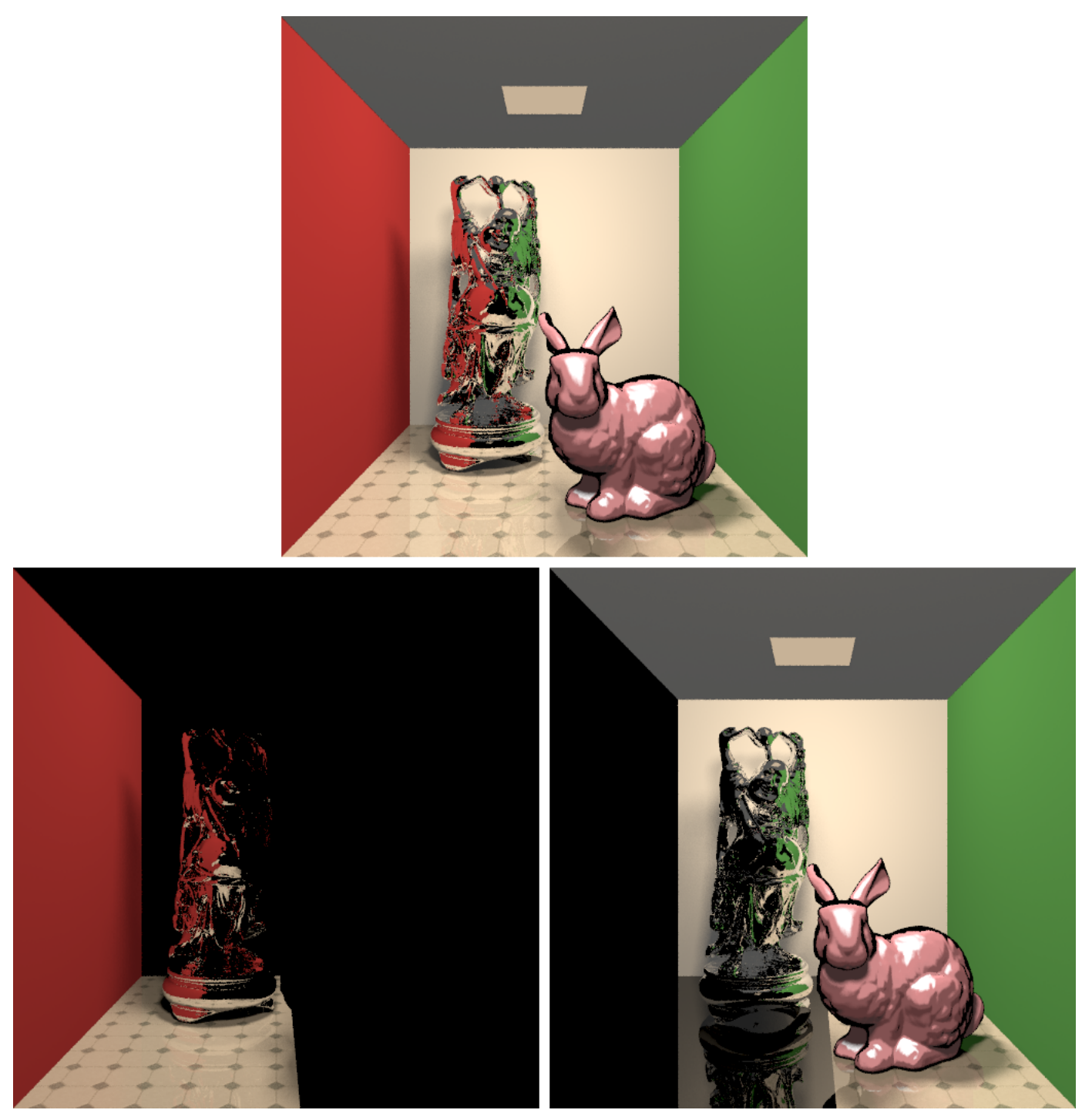

Figure 4.10: The left and right images show the geometry split between two workers. The composite image is in the center. Note how the Buddha reflects the left side geometry in the left image and the right side geometry in the right image. The actual Buddha mesh data was distributed to the worker on the left. 


\section{Chapter 5}

\section{Results}

\subsection{Experimental Setup}

For testing, we used 2-16 Dell T3500 workstations with dual-core Intel Xeon W3503 CPUs clocked at $2.4 \mathrm{GHz}$. Each workstation had $4 \mathrm{~GB}$ of system RAM, a 4 GB swap partition, and was running CentOS 6.

On the software side, FlexRender consists of around 14,000 lines of code. Of that, $80 \%$ is $\mathrm{C}++$ (with fairly extensive use of $\mathrm{C}++11$ ), $17 \%$ is Lua (scene and shader support libraries), and $3 \%$ is Bash script used for building library dependencies.

We leverage several popular open source libraries:

- LuaJIT, for a fast implementation of of the Lua programming language.

- libuv, the C library behind Node.js, for asynchronous networking and thread pools.

- MsgPack, for $\mathrm{C}++$ class serialization. 
- GLM, a C++ implementation of the GLSL math specification.

- OpenEXR, for writing full 32-bit float HDR images.

We also made use of the pfstools and pdiff software packages for tone mapping our output images and computing perceptual diffs respectively.

\subsection{Toy Store}

Our test scene, "Toy Store", has a geometric complexity of nearly 42 million triangles. It consists of $1.09 \mathrm{~GB}$ of mesh data and $5 \mathrm{~GB}$ of BVH data once the acceleration structures are built.

The room geometry is relatively simple, but the toys on the shelves are unique (non-instanced) copies of the Stanford bunny, Buddha, and dragon models that have been remeshed from their highest quality reconstructions down to 14,249 faces, 49,968 faces, and 34,972 faces respectively. There are 30 toys per shelf and 42 shelves in the scene for a total of nearly 1,300 models. Approximately one quarter of the models are rendered with a mirror shader. The others have a standard Phong shader.

The image was rendered at a resolution of 1024x768 with no subpixel antialiasing, 10 Monte Carlo samples per light (32 lights in the scene), and a recursion depth limit of 3 bounces.

\subsection{Render Time}

For discussing render time speedups, we will consider the specific case of 8 workers in the traditional configuration vs. 8 workers in the FlexRender config- 


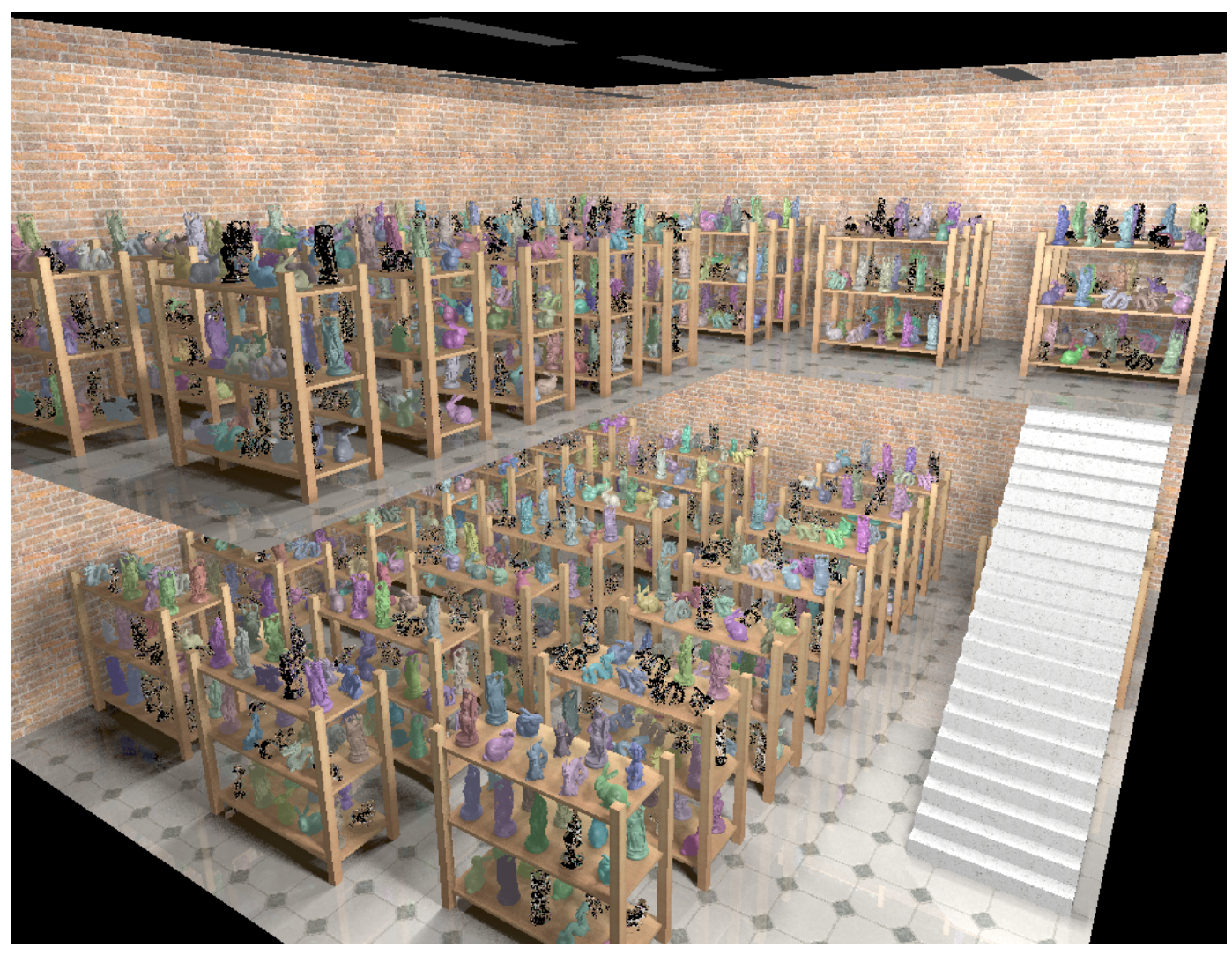

Figure 5.1: The "Toy Store" scene used for benchmarking FlexRender.

uration. We will cover other cluster sizes in less depth in Section 5.7.

\subsubsection{Traditional Method}

The traditional method works by chopping up the image into several vertical slices, with a different machine responsible for each slice. The slices are then reassembled to form the final output image.

We report time for each machine to complete each phase of rendering (loading the scene, building the BVH, and rendering its slice of the image) in Table 5.1. In particular, the total length of the render would take 10,061 seconds (the slowest time), because the last slice is needed before the final image can be reassembled. 

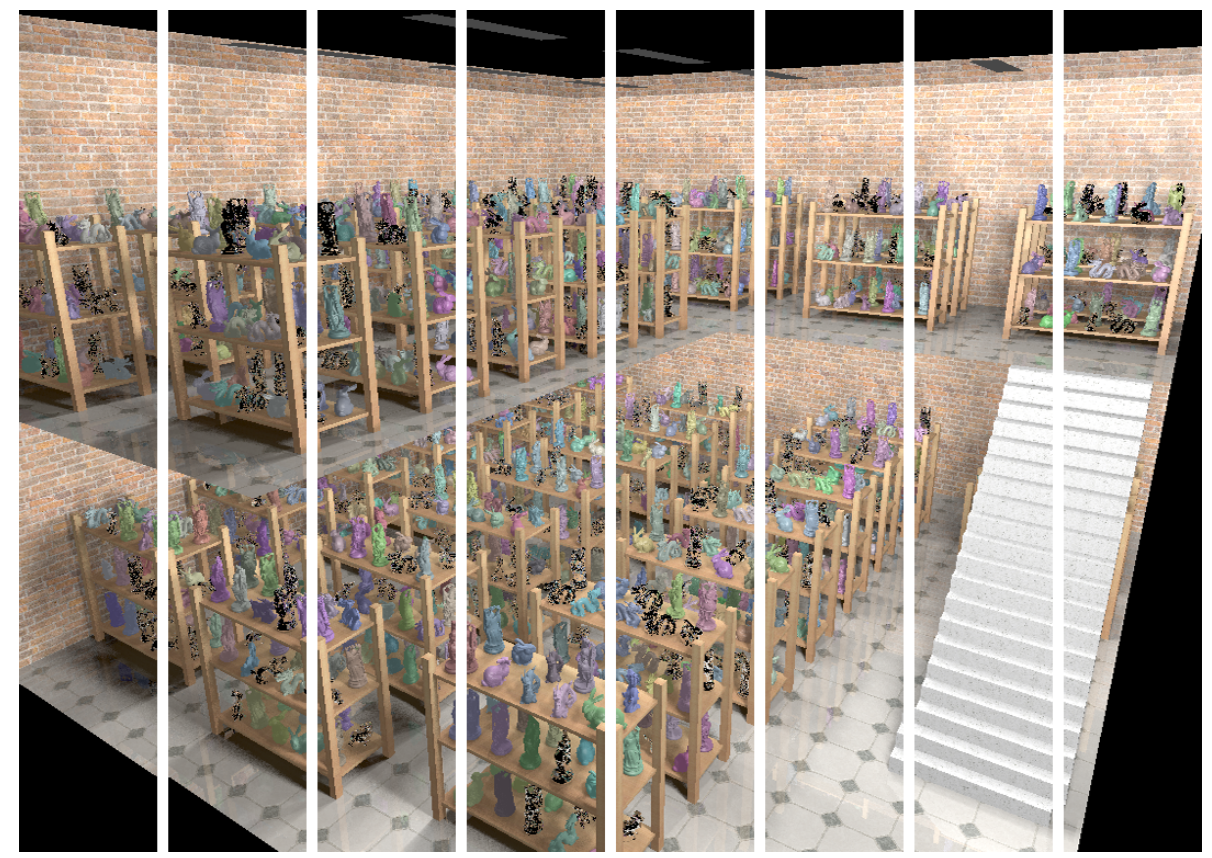

Figure 5.2: Slices of the Toy Store scene rendered with 8 machines in the traditional configuration.

\begin{tabular}{|l||c|c|c|c|c|c|c|c|}
\hline & 1 & 2 & 3 & 4 & 5 & 6 & 7 & 8 \\
\hline \hline Loading & 288 & 290 & 290 & 289 & 295 & 290 & 287 & 294 \\
\hline Building BVH & 169 & 172 & 167 & 261 & 174 & 192 & 165 & 171 \\
\hline Rendering & 533 & 1119 & 1491 & 9511 & 1792 & 677 & 1109 & 303 \\
\hline \hline Total & 990 & 1581 & 1948 & 10061 & 2261 & 1159 & 1561 & 768 \\
\hline
\end{tabular}

Table 5.1: Time (in seconds) for individual workers to complete each phase using the traditional method.

\subsubsection{FlexRender}

For comparison purposes, we report average times for the traditional workers compared to FlexRender for each of the 3 rendering phases in Table 5.2. We also show FlexRender's speedup over the average case.

We also report the total render time (all three phases combined), using this time the slowest traditional worker. This is a fair comparison because even if all of the other traditional workers finish quickly, the render is not complete until 


\begin{tabular}{|l||c|c|c|}
\hline & Traditional & FlexRender & Speedup \\
\hline \hline Loading & 290.4 (avg) & 326 & $0.89 x$ \\
\hline Building BVH & 183.6 (avg) & 20 & $9.18 \mathrm{x}$ \\
\hline Rendering & 2066.9 (avg) & 1186 & $1.74 x$ \\
\hline \hline Total & $\mathbf{1 0 0 6 1}$ (slowest) & $\mathbf{1 5 3 2}$ & $\mathbf{6 . 5 7 x}$ \\
\hline Total (w/o loading) & $\mathbf{9 8 0 0}$ (slowest) & $\mathbf{1 2 0 6}$ & $\mathbf{8 . 1 3 x}$ \\
\hline
\end{tabular}

Table 5.2: Time (in seconds) for each phase of rendering with FlexRender and the traditional configuration.

the last worker finishes.

Lastly, we also report the times and speedup excluding the loading and syncing phase. The reasoning behind this is that while FlexRender currently reads in scene assets at the renderer and distributes them across the network, there is no inherent reason why it needs to load the scene this way. Specifically, if each worker had access to a shared network storage volume with the scene assets on it, the renderer could simply issue commands to each worker instructing it to load a particular asset from the network storage.

\subsection{Geometry Distribution}

To ensure the entire scene stays in core, FlexRender must distribute the geometry across the available RAM in the cluster effectively. With the exception of one worker, the Morton coding and Z-order curve did a decent job of partitioning the scene data evenly.

The one worker which did not contain very much geometry was in the top corner of the Toy Store closest to the camera. This octant of the scene only contained a fill light facing the rest of the geometry. 


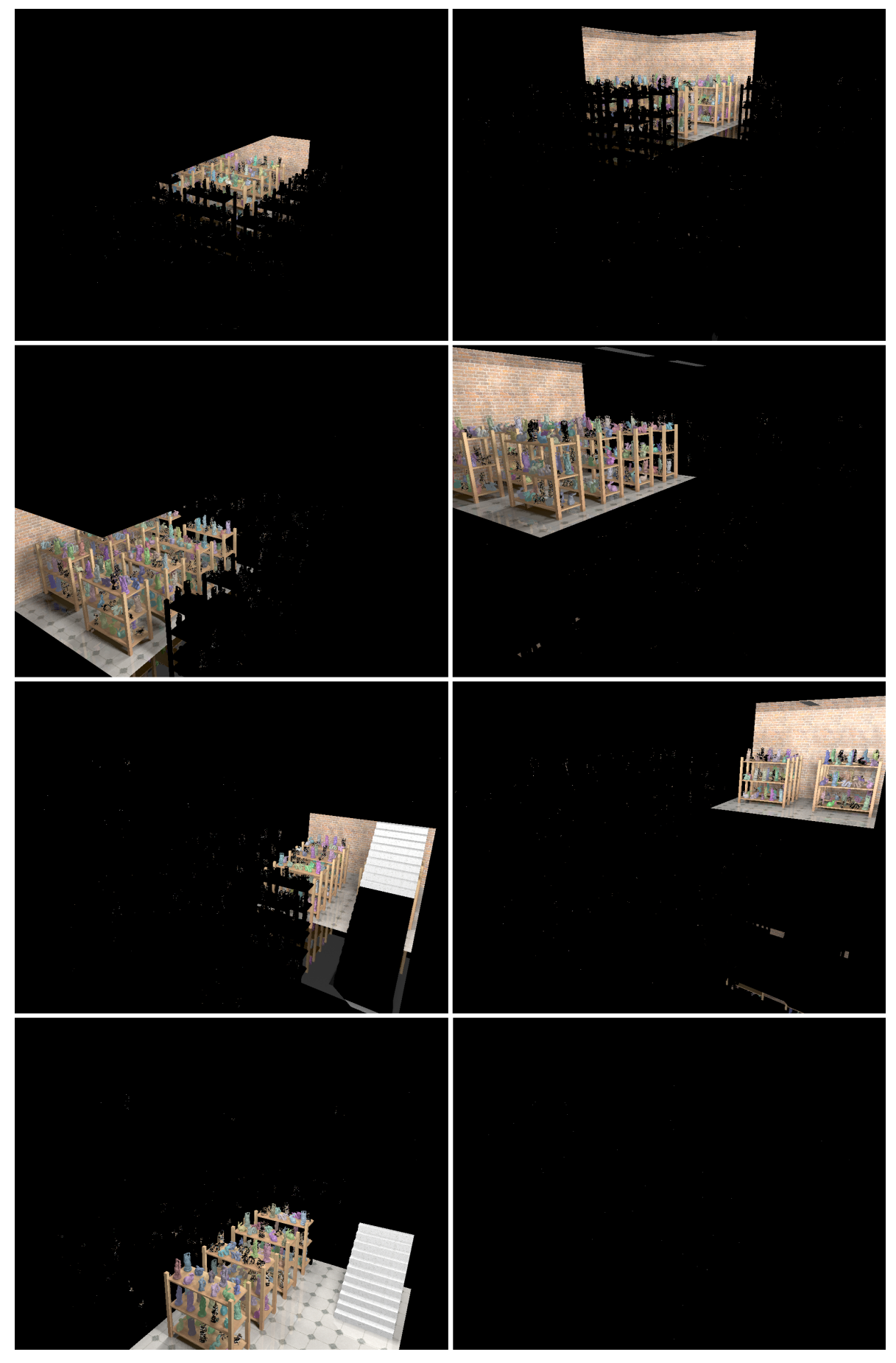

Figure 5.3: The individual worker image buffers that are combined to form the final image. 


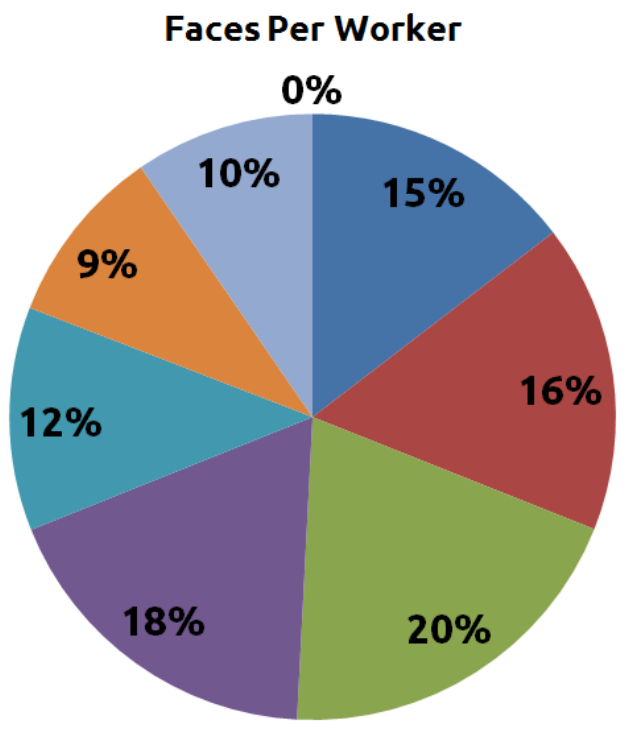

Figure 5.4: Percentage of total geometry that was distributed to each worker.

\begin{tabular}{|l||c|c|c|c|c|c|c|c|}
\hline & 1 & 2 & 3 & 4 & 5 & 6 & 7 & 8 \\
\hline \hline Geometry Size (MB) & 162 & 183 & 221 & 202 & 133 & 106 & 107 & 0 \\
\hline BVH Size (MB) & 742 & 837 & 1007 & 922 & 607 & 486 & 488 & 0 \\
\hline
\end{tabular}

Table 5.3: Distribution of geometry and acceleration structure data based on storage requirements.

\subsection{Network Hops}

The intent of the top-level BVH is to reduce network cost by only sending a ray across the network when we know it ventures into that worker's region of space. Since a BVH is a $O(\log n)$ data structure, we expect that with 8 workers the average ray would be handled by 3 workers during its lifetime.

\begin{tabular}{|r||c|c|c|c|c|c|c|c|}
\hline Num. of Workers & 1 & 2 & 3 & 4 & 5 & 6 & 7 & $8+$ \\
\hline Pct. of Rays & $15.8 \%$ & $25.6 \%$ & $21.8 \%$ & $18.8 \%$ & $8.9 \%$ & $7.4 \%$ & $1.5 \%$ & $0.2 \%$ \\
\hline
\end{tabular}

Table 5.4: Percentage of rays that were processed by the given number of workers. 
Our results show that $63.2 \%$ of rays are handled by 3 workers or less, and nearly $16 \%$ of them never even go out over the network (they are generated on, intersect with, and are shaded on a single worker). We also see that $36.8 \%$ of rays must touch more than the expected 3 workers.

However, if we include the corner cases mentioned in 4.3 .3 , we see that $82 \%$ of rays are at or below $O((\log n)+1)$ and $90.9 \%$ of rays are at or below $O((\log n)+2)$. In Section 6.1.1 we discuss some potential methods for reducing or removing these extra network hops.

\subsection{Ray Queue Sizes}

Keeping the ray queue size small is critical to the long term health of the render. If rays begin piling up faster than the cluster can process them, eventually the cluster will begin swapping when accessing rays, which violates our fundamental performance goal to stay off of disk.

Because of this, we benchmarked the ray queue sizes on each worker over time when rendering Toy Store on a cluster of 16 workers. Figure 5.5 shows this with each renderer displayed as a different color. Table 5.5 breaks down the average

queue size, as well as the maximum size over the course of the entire render and the storage requirements of the maximum size, both in terms of raw storage space and a fraction of the system RAM.

As Table 5.5 shows, most workers sit comfortably below $1 \%$ of their RAM being used for queued rays, while the busiest worker used just over 1\%. This demonstrates that our regulation mechanisms and work throttling is working well to keep the cluster from generating more work than it can handle. 


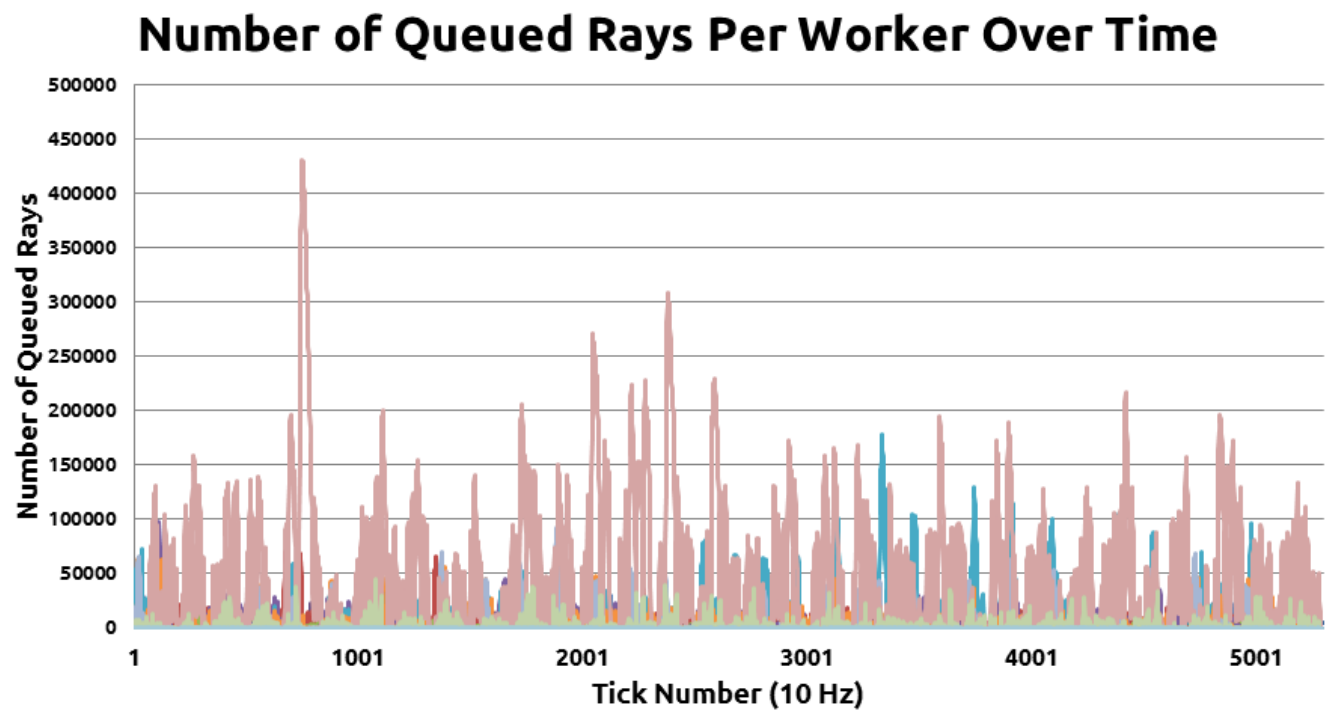

Figure 5.5: Number of rays queued on each worker over time. Different colors correspond to different workers.

\subsection{Cluster Size}

To evaluate the scalability of the FlexRender architecture, we ran Toy Store renders with cluster sizes of 4 workers, 8 workers, and 16 workers. For comparison, we ran the same renders in the traditional configuration also of 4,8 , and 16 workers.

As Table 5.6 shows, we see a continual and impressive improvement in the BVH construction time as the number of workers increases. This is thanks to the huge parallelization speedup we get from partitioning the scene geometry with Morton coding and building each subtree in parallel.

We also see render time improvements of roughly an order of magnitude, depending on how FlexRender chooses to distribute the geometry based on the number of workers available. It is likely that this performance advantage will slowly erode as the cluster size grows larger (due to increasing network communication costs), but for small to medium cluster sizes we see approximately linear 


\begin{tabular}{|c||c|c|c|c|}
\hline Worker & Average Queued & Max Queued & Max Storage & Memory Occupancy \\
\hline \hline 1 & 107 & 12221 & $1.49 \mathrm{MB}$ & $0.04 \%$ \\
\hline 2 & 506 & 29796 & $3.64 \mathrm{MB}$ & $0.09 \%$ \\
\hline 3 & 969 & 34970 & $4.27 \mathrm{MB}$ & $0.10 \%$ \\
\hline 4 & 3116 & 57597 & $7.03 \mathrm{MB}$ & $0.17 \%$ \\
\hline 5 & 1687 & 36981 & $4.51 \mathrm{MB}$ & $0.11 \%$ \\
\hline 6 & 1430 & 44053 & $5.38 \mathrm{MB}$ & $0.13 \%$ \\
\hline 7 & 4070 & 80899 & $9.88 \mathrm{MB}$ & $0.24 \%$ \\
\hline 8 & 2000 & 16861 & $2.06 \mathrm{MB}$ & $0.05 \%$ \\
\hline 9 & 3767 & 97429 & $11.89 \mathrm{MB}$ & $0.29 \%$ \\
\hline 10 & 7921 & 178191 & $21.75 \mathrm{MB}$ & $0.53 \%$ \\
\hline 11 & 4477 & 69702 & $8.51 \mathrm{MB}$ & $0.21 \%$ \\
\hline 12 & 4943 & 92043 & $11.24 \mathrm{MB}$ & $0.27 \%$ \\
\hline 13 & 51164 & 430736 & $52.58 \mathrm{MB}$ & $1.28 \%$ \\
\hline 14 & 2193 & 45428 & $5.55 \mathrm{MB}$ & $0.14 \%$ \\
\hline 15 & 0 & 0 & $0 \mathrm{MB}$ & $0.00 \%$ \\
\hline 16 & 18 & 1374 & $0.17 \mathrm{MB}$ & $0.01 \%$ \\
\hline
\end{tabular}

Table 5.5: Size of ray queues when rendering Toy Store with a 16worker FlexRender cluster.

growth.

\subsection{Example Renders}

Figure 5.6 shows the final Toy Store scene render, with 42 million triangles and nearly 1,300 models.

Figure 5.7 shows a render with even higher resolution models, bringing the geometric complexity up to 87 million triangles.

Figure 5.8 shows a monochromatic Cornell Box rendered by two workers. The final composite is on the left, while the individual image buffers are on the right. With only direct lighting and no reflections, this clearly demonstrates which geometry was assigned to each worker. 


\begin{tabular}{|l||c|c|c|}
\hline & 4 Workers & 8 Workers & 16 Workers \\
\hline \hline BVH Build (trad.) & $203 \mathrm{sec}$ & $184 \mathrm{sec}$ & $171 \mathrm{sec}$ \\
\hline BVH Build (flex.) & $39 \mathrm{sec}$ & $20 \mathrm{sec}$ & $10 \mathrm{sec}$ \\
\hline BVH Build (speedup) & $\mathbf{5 . 2 1 x}$ & $\mathbf{9 . 2 0 x}$ & $\mathbf{1 7 . 1 x}$ \\
\hline \hline Total Render (trad.) & $14833 \mathrm{sec}$ & $10061 \mathrm{sec}$ & $7702 \mathrm{sec}$ \\
\hline Total Render (flex.) & $1742 \mathrm{sec}$ & $1532 \mathrm{sec}$ & $970 \mathrm{sec}$ \\
\hline Total Render (speedup) & $\mathbf{8 . 5 1 x}$ & $\mathbf{6 . 5 7 x}$ & $\mathbf{7 . 9 4 x}$ \\
\hline \hline Render w/o Load (trad.) & $14536 \mathrm{sec}$ & $9800 \mathrm{sec}$ & $7413 \mathrm{sec}$ \\
\hline Render w/o Load (flex.) & $1405 \mathrm{sec}$ & $1206 \mathrm{sec}$ & $540 \mathrm{sec}$ \\
\hline Render w/o Load (speedup) & $\mathbf{1 0 . 3 5 x}$ & $\mathbf{8 . 1 3 x}$ & $\mathbf{1 3 . 7 3 x}$ \\
\hline
\end{tabular}

Table 5.6: Comparison of cluster sizes with both the traditional configuration and the FlexRender configuration.

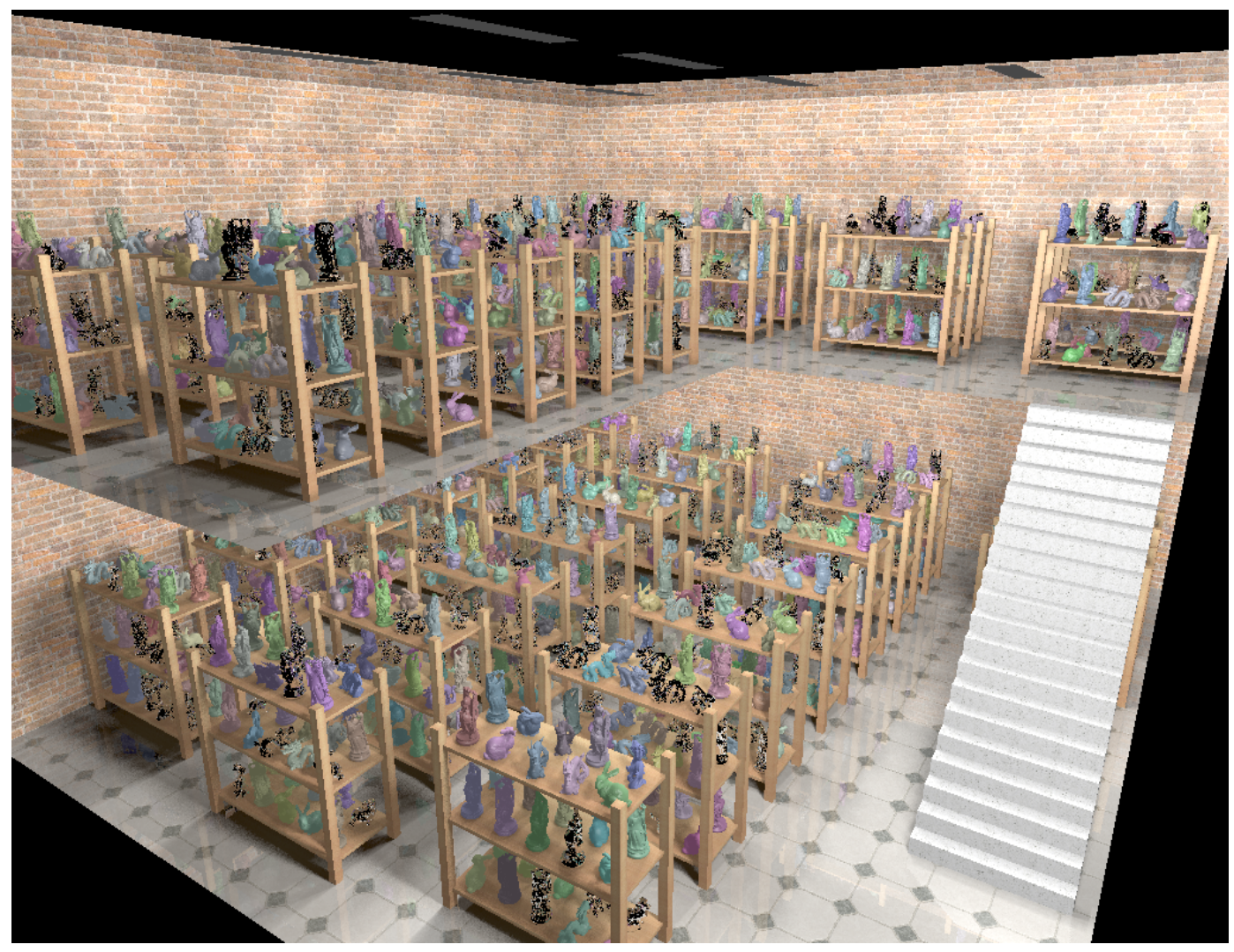

Figure 5.6: Toy Store scene that is comprised of nearly 1,300 models and 42 million triangles.

Figure 5.9 demonstrates FlexRender's Lua-based shader system. The Stanford bunny model is toon shaded, while the Buddha model has a perfect mirror 


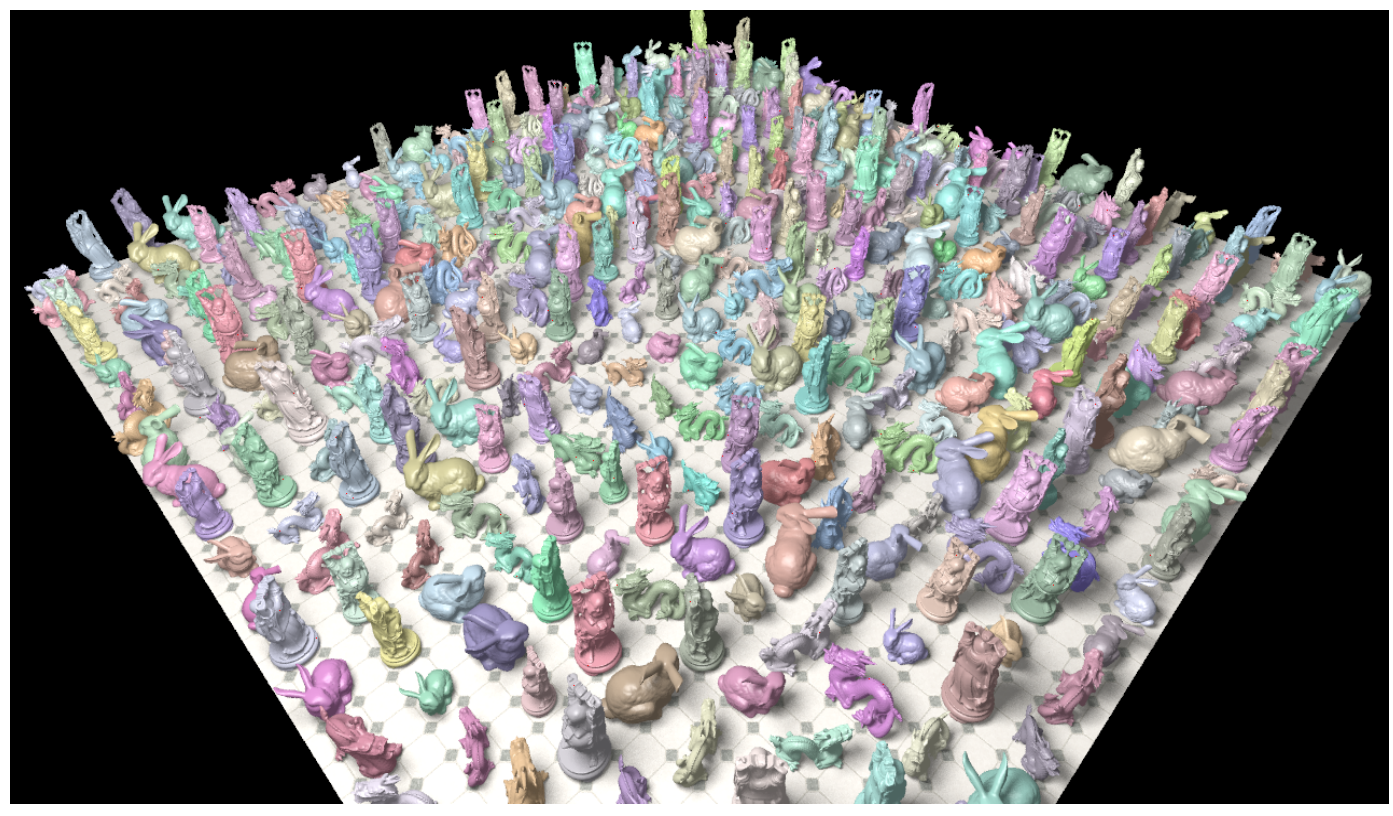

Figure 5.7: Field of high resolution models with nearly 87 million triangles.

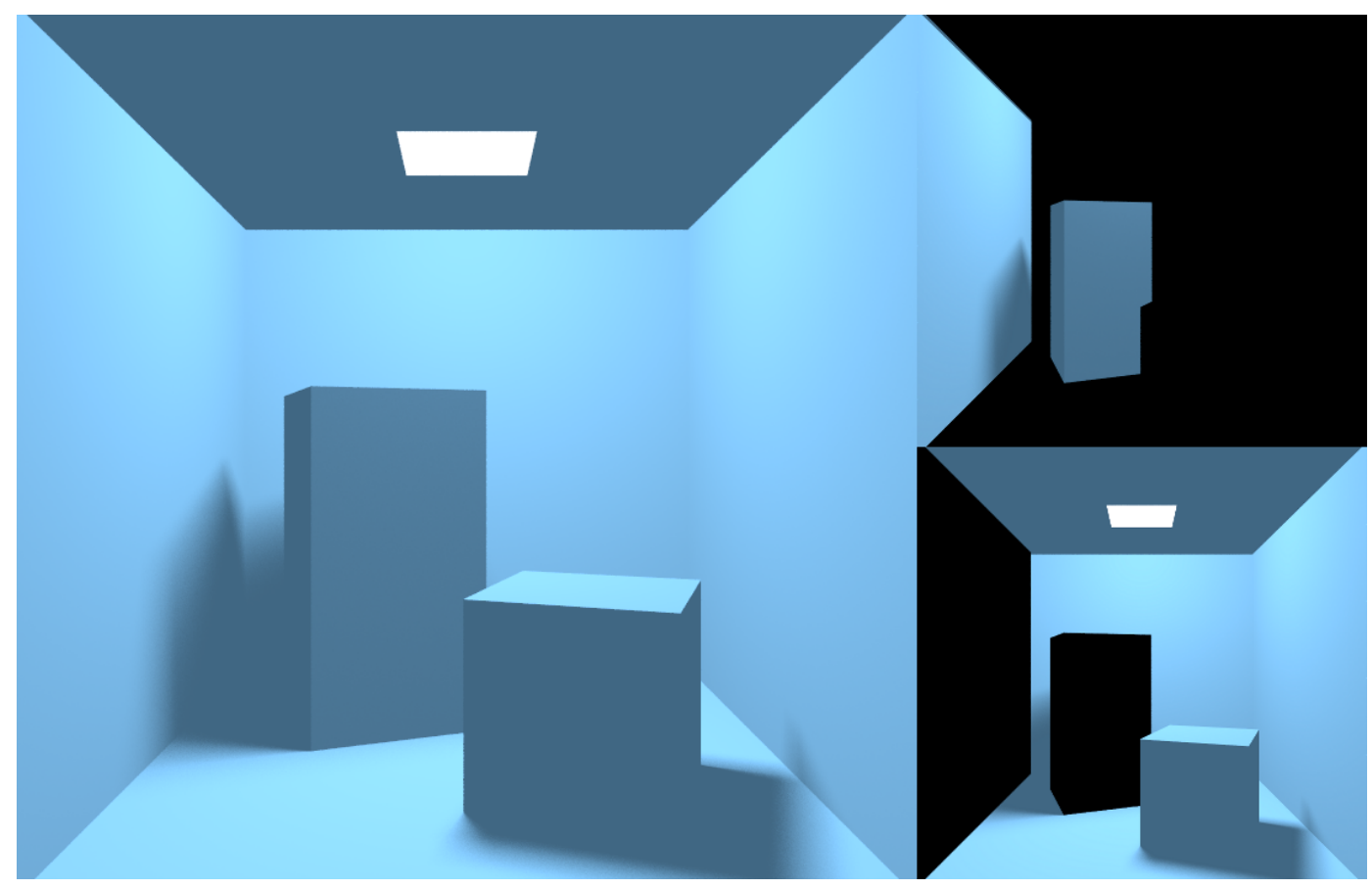

Figure 5.8: A monochromatic Cornell Box, showing the distribution of geometry between two workers. 
finish. Reflection rays for the mirrored Buddha and floor are cast directly from the shaders.

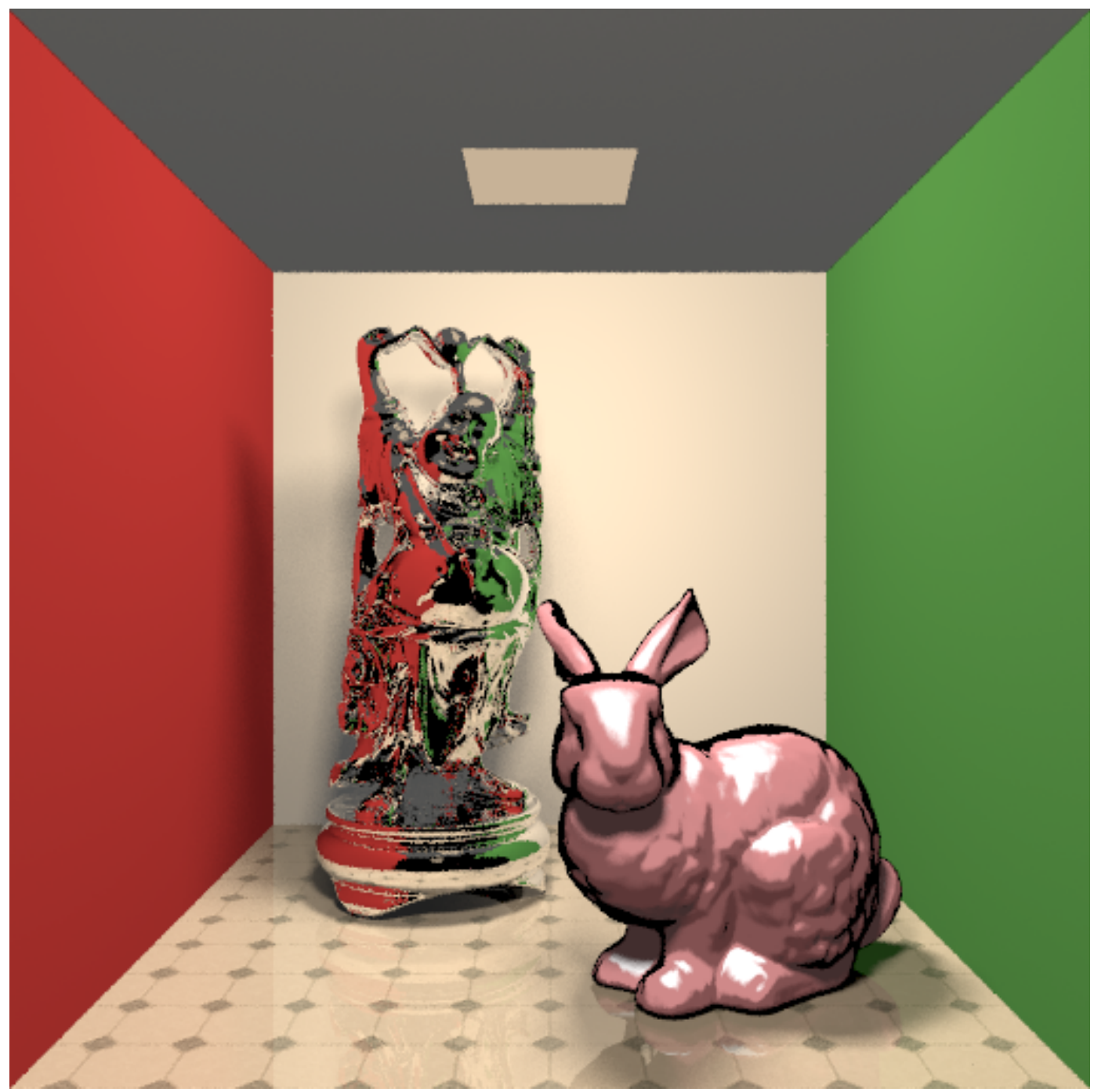

Figure 5.9: A Cornell Box with a toon shaded Stanford bunny and mirrored Buddha.

Figure 5.10 shows an example of a Monte Carlo global illumination shader, as rendered by two workers. At first it appears that the geometry is present on all workers. However, the lighting tells the full story. The geometry is split the same way as in Figure 5.8. Each worker image contains direct light for the geometry on that worker, as well as indirect light reflected by geometry on that worker. 


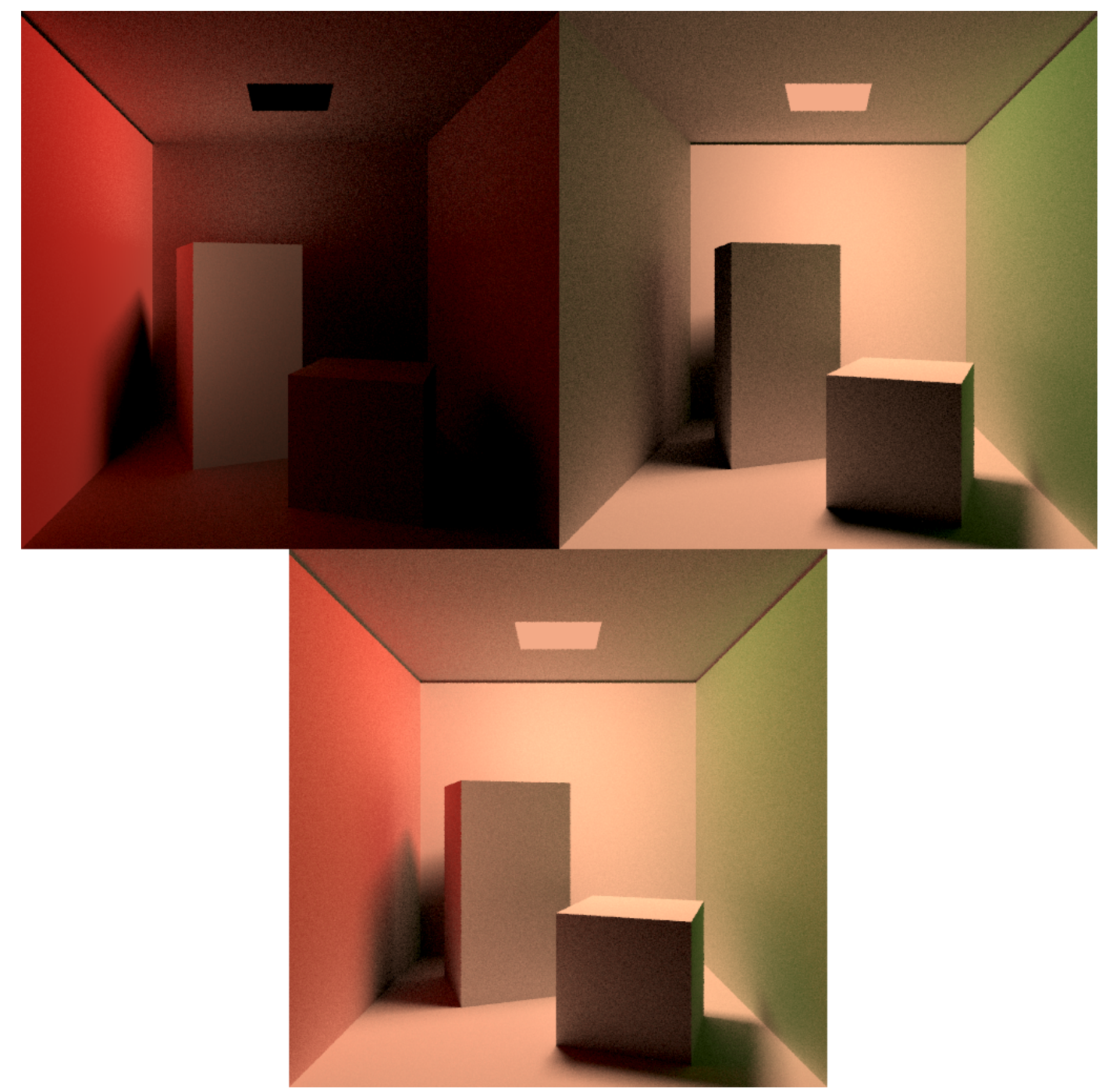

Figure 5.10: A Cornell Box with a Monte Carlo global illumination shader. The top row shows individual worker renders, the bottom center is the final composite. Each worker has direct light for the geometry on its machine and indirect light caused by the geometry on its machine.

\subsection{Summary}

With these results, we have demonstrated that FlexRender meets our claimed contributions in the following ways.

- The scene geometry and acceleration structure data stays in-core due to 
effective scene distribution.

- Rays carry enough state to never require replies and the generated images match our baseline implementation.

- We can correctly and efficiently traverse the scene when workers only contain spatially local BVHs, linked by a top-level BVH.

- The top-level BVH effectively reduces the amount of network communication necessary to render the scene.

- The system keeps itself regulated and reduces memory usage by throttling new work generation and processing rays in an intelligent order.

- We show consistent speedups over the traditional approach, which suffers greatly from having to hit disk frequently during the rendering process. 


\section{Chapter 6}

\section{Future Work}

Given that FlexRender is a relatively radical reimagining of ray tracer architecture, the opportunities for improvement are vast. In this section we document larger issues we noticed during implementation and testing that could potentially offer significantly better results.

\subsection{System Optimizations}

We present several potential optimizations. In Section 6.1.1 we discuss a potential method to deter or eliminate the extra network hops in BVH traversal, and in Section 6.1.2 we discuss some underlying issues that, if addressed, would yield better geometry distribution and parallelism.

\subsubsection{Eliminating Extra Network Hops}

The first extra network hop occurs when rays are generated on machines that control geometry they do not intersect with. This requires at least one network 
hop before the ray could potentially intersect and be shaded. By performing a prepass step before rendering began, we could gather information about the layout of the scene in image space. By carefully choosing the slices of the image that workers were responsible for casting primary rays in, we could dramatically increase the chances that the ray would intersect the geometry on the worker and finish shading without ever touching the network.

The second extra network hop occurs at the end of traversal, when the ray finishes traversing the top-level BVH but ends up on a worker that is not the point of intersection. Because the worker has no shading assets for that geometry, it must pass the ray back to the "winning" worker for shading. By distributing all of the shading assets (shaders, textures, and materials) to all workers, any worker would be capable of shading a ray at the conclusion of its BVH traversal. This is possible because the rays carry along local geometry (mainly the surface normal) from the point of closest intersection.

\subsubsection{Competing Concerns}

Currently there are two competing concerns in FlexRender. The first is to distribute the scene data well, such that the geometry distribution is balanced and everything is in-core. The second is to "keep the chips hot", or maximize the utilization of the available processing power in the cluster. Unfortunately, these concerns are somewhat at odds at the moment. Good geometry distribution is a result of distributing 3D space well, whereas good workload distribution is a result of distributing screen space well. It is not immediately clear if these two competing concerns can be addressed by a single renderer, but we believe significant improvements could be made to both the geometry distribution and 
the workload distribution that would significantly improve the state of affairs.

In retrospect, we believe that the Morton coding and Z-order curve was a poor choice for the distribution of geometry. It was initially chosen because we did not want to require a preprocessing (or baking) step for determining the optimal distribution of geometry. Since the scene was presumably too large to fit in core on any one machine, preprocessing the scene as a whole was deemed to be against the spirit of the problem.

The Z-order curve works well for spatially uniform scenes, but unfortunately a lot of scenes that are interesting to render are usually uniformly distributed in image space, not 3D space. The leads to a lot of degenerate scenes that perform suboptimally when rendered with FlexRender. There would be a lot more opportunities for distributing geometry in a more intelligent way if a preprocessing step was allowed.

Secondly, the workload of rendering a particular piece of geometry falls on the machine who is responsible for that geometry. This means that if a single piece of geometry takes up a large portion of screen space, it will end up being the bottleneck in rendering. Conversely, workers that hold geometry that takes up very little screen space could end up contributing very little horsepower to the render.

As mentioned in Section 6.1.1, it is possible that a quick prepass rendering could help alleviate this problem. If we have information about the layout of the scene in image space prior to rendering, we have some opportunities for moving things around to adjust the workload before it becomes a problem during rendering. It is also possible that additional statistics monitoring could provide on-the-fly migration of scene data to less loaded workers, although that would 
require at least partially rebuilding the acceleration structure.

\subsection{Memory Optimizations}

Based on the profiling results of our current implementation, the main bottleneck is standard library calls and memory allocation. The transient nature of many fixed-size fat rays is a perfect opportunity to use an object pool to reduce allocation overhead and heap fragmentation.

In addition, the linear BVH nodes structures were intentionally padded to 64bytes to match the cache line size on current CPUs. However without a custom STL allocator for the vector that stores this array of nodes, cache alignment is not guaranteed.

Lastly, there are a few places where the size of fat rays could be reduced. For example, rather than storing the target position of an expected intersection, the expected $t$ value along the ray could be stored instead. This would save 8 bytes. Another 4 bytes could be saved by removing the "workers touched" benchmarking field. By careful overlapping of mutually exclusive data using unions, the size could probably be reduced further. Ideally a fat ray would fit on a single cache line and the same aligned allocation strategy could be applied to fat rays as well.

\subsection{GPGPU and Heterogeneous Architectures}

One of FlexRender's strengths is that it decouples the ray tracing computations at the level of the individual ray. Because workers in the cluster speak a 
defined network protocol to pass rays around, this opens the door for workers to join the cluster with new and interesting machine architectures.

For example, consider general purpose GPU (GPGPU) computing. Vast speedups can be attained by moving number-crunching operations to the GPU, but the CPU and GPU usually exist in separate memory spaces. This means the memory transfer cost of pushing data over the bus to the GPU must be amortized by the amount of computation done on the GPU.

Because workers are never waiting on other workers for the results of a computation, this opens up the possibility for batching data to a GPU worker. Consider a worker who simply queues messages until enough are present to move the data to the the GPU. FlexRender's architecture is amiable to amortizing the cost of bus transfers in GPGPU computing. 


\section{Bibliography}

[1] Arthur Appel. Some techniques for shading machine renderings of solids. In Spring Joint Comptr. Conf., AFIPS, pages 37-45, 1968.

[2] P. Cignoni, C. Montani, R. Scopigno, and C. Rocchini. A general method for preserving attribute values on simplified meshes. In Proceedings of the conference on Visualization '98, VIS '98, pages 59-66, Los Alamitos, CA, USA, 1998. IEEE Computer Society Press.

[3] James H. Clark. Hierarchical geometric models for visible-surface algorithms. In Proceedings of the 3rd annual conference on Computer graphics and interactive techniques, SIGGRAPH '76, pages 267-267, New York, NY, USA, 1976. ACM.

[4] Jonathan Cohen, Marc Olano, and Dinesh Manocha. Appearance-preserving simplification. In Proceedings of the 25th annual conference on Computer graphics and interactive techniques, SIGGRAPH '98, pages 115-122, New York, NY, USA, 1998. ACM.

[5] Jeffrey Dean and Sanjay Ghemawat. Mapreduce: simplified data processing on large clusters. In Proceedings of the 6th conference on Symposium on Opearting Systems Design 83 Implementation - Volume 6, OSDI'04, pages 10-10, Berkeley, CA, USA, 2004. USENIX Association. 
[6] Kirill Garanzha, Alexander Bely, Simon Premoze, and Vladimir Galaktionov. Out-of-core gpu ray tracing of complex scenes. In ACM SIGGRAPH 2011 Talks, SIGGRAPH '11, pages 21:1-21:1, New York, NY, USA, 2011. ACM.

[7] Kirill Garanzha, Simon Premoze, Alexander Bely, and Vladimir Galaktionov. Grid-based sah bvh construction on a gpu. Vis. Comput., 27(68):697-706, June 2011.

[8] M. Hapala, T. Davidovic, I. Wald, V. Havran, and P. Slusallek. Efficient stack-less bvh traversal for ray tracing. In 27th Spring Conference on Computer Graphics, SCCG' '11, 2011.

[9] Toshi Kato and Jun Saito. "kilauea": parallel global illumination renderer. In Proceedings of the Fourth Eurographics Workshop on Parallel Graphics and Visualization, EGPGV '02, pages 7-16, Aire-la-Ville, Switzerland, Switzerland, 2002. Eurographics Association.

[10] Janne Kontkanen, Eric Tabellion, and Ryan S. Overbeck. Coherent out-ofcore point-based global illumination. In Eurographics Symposium on Rendering, 2011.

[11] Venkat Krishnamurthy and Marc Levoy. Fitting smooth surfaces to dense polygon meshes. In Proceedings of the 23rd annual conference on Computer graphics and interactive techniques, SIGGRAPH '96, pages 313-324, New York, NY, USA, 1996. ACM.

[12] The Lua programming language. http://www.lua.org.

[13] The LuaJIT project. http://luajit.org. 
[14] Lesley Northam and Rob Smits. Hort: Hadoop online ray tracing with mapreduce. In ACM SIGGRAPH 2011 Posters, SIGGRAPH '11, pages 22:1-22:1, New York, NY, USA, 2011. ACM.

[15] Jacopo Pantaleoni, Luca Fascione, Martin Hill, and Timo Aila. Pantaray: fast ray-traced occlusion caching of massive scenes. In ACM SIGGRAPH 2010 papers, SIGGRAPH '10, pages 37:1-37:10, New York, NY, USA, 2010. ACM.

[16] Matt Pharr and Greg Humphreys. Physically Based Rendering: From Theory to Implementation. Morgan Kaufmann, 2nd edition, 2010.

[17] Turner Whitted. An improved illumination model for shaded display. Commun. ACM, 23(6):343-349, June 1980.

[18] Zhefeng Wu, Fukai Zhao, and Xinguo Liu. Sah kd-tree construction on gpu. In Proceedings of the ACM SIGGRAPH Symposium on High Performance Graphics, HPG '11, pages 71-78, New York, NY, USA, 2011. ACM. 